\title{
Higher Genus Characters for Vertex Operator Superalgebras on Sewn Riemann Surfaces
}

\author{
Alexander Zuevsky \\ Max-Planck-Institut für Mathematik, Bonn, Germany \\ Email: zuevsky@mpim-bonn.mpg.de
}

Received May 21, 2013; revised June 21, 2013; accepted June 28, 2013

Copyright (C) 2013 Alexander Zuevsky. This is an open access article distributed under the Creative Commons Attribution License, which permits unrestricted use, distribution, and reproduction in any medium, provided the original work is properly cited.

\begin{abstract}
We review our recent results on computation of the higher genus characters for vertex operator superalgebras modules. The vertex operator formal parameters are associated to local parameters on Riemann surfaces formed in one of two schemes of (self- or tori-) sewing of lower genus Riemann surfaces. For the free fermion vertex operator superalgebra we present a closed formula for the genus two continuous orbifold partition functions (in either sewings) in terms of an infinite dimensional determinant with entries arising from the original torus Szegö kernel. This partition function is holomorphic in the sewing parameters on a given suitable domain and possesses natural modular properties. Several higher genus generalizations of classical (including Fay's and Jacobi triple product) identities show up in a natural way in the vertex operator algebra approach.
\end{abstract}

Keywords: Vertex Operator Superalgebras; Intertwining Operators; Riemann Surfaces; Szegö Kernel; Modular Forms; Theta-Functions; Frobenius-Fay and Jacobi Product Identities

\section{Vertex Operator Superalgebras}

In this paper (based on several conference talks of the author) we review our recent results [1-5] on construction and computation of correlation functions of vertex operator superalgebras with a formal parameter associated to local coordinates on a self-sewn Riemann surface of genus $g$ which forms a genus $g+1$ surface. In particular, we review result presented in the papers [1-5] accomplished in collaboration with M. P. Tuite (National University of Ireland, Galway, Ireland).

A Vertex Operator Superalgebra (VOSA) [6-10] is a quadruple $(V, Y, \mathbf{1}, \omega)$ :

$$
V=V_{\overline{0}} \oplus V_{\overline{1}}=\bigoplus_{r \in \frac{1}{2} \mathbb{Z}} V_{r},
$$

$\operatorname{dim} V_{r}<\infty$, is a superspace, $Y$ is a linear map

$$
Y: V \rightarrow(\operatorname{End} V)\left[\left[z, z^{-1}\right]\right],
$$

so that for any vector (state) $u \in V \quad$ we have

$$
\begin{aligned}
& u(k) \mathbf{1}=\delta_{k,-1} u, \quad k \geq-1, \\
& Y(u, z)=\sum_{n \in \mathbb{Z}} u(n) z^{-n-1}, \\
& u(n) V_{\alpha} \subset V_{\alpha+p(u)}, \quad p(u) \text {-parity. }
\end{aligned}
$$

The linear operators (modes) $u(n): V \rightarrow V$ satisfy creativity

$$
Y(u, z) \mathbf{1}=u+O(z),
$$

and lower truncation

$$
u(n) v=0,
$$

conditions for $u, v \in V$ and $n \gg 0$.

These axioms identity impy locality, associativity, commutation and skew-symmetry:

$$
\begin{aligned}
& \left(z_{1}-z_{2}\right)^{m} Y\left(u, z_{1}\right) Y\left(v, z_{2}\right) \\
& =(-1)^{p(u, v)}\left(z_{1}-z_{2}\right)^{m} Y\left(v, z_{2}\right) Y\left(u, z_{1}\right), \\
& \left(z_{0}+z_{2}\right)^{n} Y\left(u, z_{0}+z_{2}\right) Y\left(v, z_{2}\right) w \\
& =\left(z_{0}+z_{2}\right)^{n} Y\left(Y\left(u, z_{0}\right) v, z_{2}\right) w, \\
& u(k) Y(v, z)-(-1)^{p(u, v)} Y(v, z) u(k) \\
& =\sum_{j \geq 0}\left(\begin{array}{c}
k \\
j
\end{array}\right) Y(u(j) v, z) z^{k-j}, \\
& Y(u, z) v=(-1)^{p(u, v)} \mathrm{e}^{z L(-1)} Y(v,-z) u,
\end{aligned}
$$

for $u, v, w \in V$ and integers $m, n \gg 0$, 


$$
p(u, v)=p(u) p(v) .
$$

The vacuum vector $\mathbf{1} \in V_{\overline{0}, 0}$ is such that, $Y(\mathbf{1}, z)=I d_{V}$, and $\omega \in V_{\overline{0}, 2}$ the conformal vector satisfies

$$
Y(\omega, z)=\sum_{n \in \mathbb{Z}} L(n) z^{-n-2},
$$

where $L(n)$ form a Virasoro algebra for a central charge $C$

$$
L(m), L(n)=(m-n) L(m+n)+\frac{C}{12}\left(m^{3}-m\right) \delta_{m,-n} .
$$

$L(-1)$ satisfies the translation property

$$
Y(L(-1) u, z)=\partial_{z} Y(u, z) \text {. }
$$

$L(0)$ describes a grading with

$$
L(0) u=w t(u) u \text {, and } V_{r}=\{u \in V \mid w t(u)=r\} \text {. }
$$

\subsection{VOSA Modules}

Definition 1 A $V$-module for a VOSA $V$ is a pair $\left(W, Y_{W}\right), W$ is a $\mathbb{C}$-graded vector space $W=\bigoplus W_{r}$, $\operatorname{dim} W_{r}<\infty, W_{r+n}=0$ for all $r$ and $n \ll 0$.

$$
\begin{gathered}
Y_{W}: V \rightarrow \operatorname{End}(W)\left[\left[z, z^{-1}\right]\right], \\
Y_{W}(u, z)=\sum_{n \in \mathbb{Z}} u_{W}(n) z^{-n-1},
\end{gathered}
$$

for each $u \in V, u_{W}: W \rightarrow W . Y_{W}(\mathbf{1}, z)=\operatorname{Id}_{W}$, and for the conformal vector

$$
Y_{W}(\omega, z)=\sum_{n \in \mathbb{Z}} L_{W}(n) z^{-n-2},
$$

where $L_{W}(0) w=r w, w \in W_{r}$. The module vertex operators satisfy the Jacobi identity:

$$
\begin{aligned}
& z_{0}^{-1} \delta\left(\frac{z_{1}-z_{2}}{z_{0}}\right) Y_{W}\left(u, z_{1}\right) Y_{W}\left(v, z_{2}\right) \\
& -(-1)^{p(u, v)} \delta\left(\frac{z_{2}-z_{1}}{-z_{0}}\right) Y_{W}\left(v, z_{2}\right) Y_{W}\left(u, z_{1}\right) \\
& =z_{2}^{-1} \delta\left(\frac{z_{1}-z_{0}}{z_{2}}\right) Y_{W}\left(Y\left(u, z_{0}\right) v, z_{2}\right) .
\end{aligned}
$$

Recall that $\delta(z)=\sum_{n \in \mathbb{Z}} z^{n}$. The above axioms imply that $L_{W}(n)$ satisfies the Virasoro algebra for the same central charge $C$ and that the translation property

$$
Y_{W}(L(-1) u, z)=\partial_{z} Y_{W}(u, z) .
$$

\subsection{Twisted Modules}

We next define the notion of a twisted $V$-module [8,11]. Let $g$ be a $V$-automorphism $g$, i.e., a linear map preserving 1 and $\omega$ such that

$$
g Y(v, z) g^{-1}=Y(g v, z),
$$

for all $v \in V$. We assume that $V$ can be decomposed into $g$-eigenspaces

$$
V=\oplus_{\rho \in \mathbb{C}} V^{\rho},
$$

where $V^{\rho}$ denotes the eigenspace of $g$ with eigenvalue $\mathrm{e}^{2 \pi i \rho}$.

Definition 2 A $g$-twisted $V$-module for a VOSA $V$ is a pair $\left(W^{g}, Y_{g}\right), \quad W^{g}=\bigoplus_{r \in \mathbb{C}} W_{r}^{g}, \quad \operatorname{dim} W_{r}^{g}<\infty$, $W_{r+n}^{g}=0$, for all $r$, and $n \ll 0 . Y_{g}: V \rightarrow$ End $W^{g}\{z\}$, the vector space of ( End $W^{g}$ )-valued formal series in $Z$ with arbitrary complex powers of $z$. For $v \in V^{\rho}$

$$
Y_{g}(v, z)=\sum_{n \in \rho+\mathbb{Z}} v_{g}(n) z^{-n-1}
$$

with $\quad v_{g}(\rho+l) w=0, \quad w \in W^{g}, \quad l \in \mathbb{Z} \quad$ sufficiently large.

$$
Y_{g}(\mathbf{1}, z)=\mathrm{Id}_{W^{g}}, Y_{g}(\omega, z)=\sum_{n \in \mathbb{Z}} L_{g}(n) z^{-n-2},
$$

where $L_{g}(0) w=r w, w \in W_{r}^{g}$. The $g$-twisted vertex operators satisfy the twisted Jacobi identity:

$$
\begin{aligned}
& z_{0}^{-1} \delta\left(\frac{z_{1}-z_{2}}{z_{0}}\right) Y_{g}\left(u, z_{1}\right) Y_{g}\left(v, z_{2}\right) \\
& -(-1)^{p(u, v)} z_{0}^{-1} \delta\left(\frac{z_{2}-z_{1}}{-z_{0}}\right) Y_{g}\left(v, z_{2}\right) Y_{g}\left(u, z_{1}\right) \\
& =z_{2}^{-1}\left(\frac{z_{1}-z_{0}}{-z_{2}}\right)^{-\rho} \delta\left(\frac{z_{1}-z_{0}}{-z_{2}}\right) Y_{g}\left(Y\left(u, z_{0}\right) v, z_{2}\right),
\end{aligned}
$$

for $u \in V^{\rho}$.

\subsection{Creative Intertwining Operators}

We define the notion of creative intertwining operators in [3]. Suppose we have a VOA $V$ with a $V$-module $\left(W, Y_{W}\right)$.

Definition 3 A Creative Intertwining Vertex Operator $\mathcal{Y}$ for a VOA $V$-module $\left(W, Y_{W}\right)$ is defined by a linear map

$$
\mathcal{Y}(w, z)=\sum_{n \in \mathbb{Z}} w(n) z^{-n-1},
$$

for $w \in W$ with modes $w(n): V \rightarrow W$; satisfies creativity

$$
\mathcal{Y}(w, z) \mathbf{1}=w+O(z),
$$

for $w \in W$ and lower truncation

$$
w(n) v=0
$$

for $v \in V, w \in W$ and $n \gg 0$. The intertwining vertex operators satisfy the Jacobi identity: 


$$
\begin{aligned}
& z_{0}^{-1} \delta\left(\frac{z_{1}-z_{2}}{z_{0}}\right) Y_{W}\left(u, z_{1}\right) \mathcal{Y}\left(w, z_{2}\right) \\
& -z_{0}^{-1} \delta\left(\frac{z_{2}-z_{1}}{-z_{0}}\right) \mathcal{Y}\left(w, z_{2}\right) Y\left(u, z_{1}\right) \\
& =z_{2}^{-1} \delta\left(\frac{z_{1}-z_{0}}{z_{2}}\right) \mathcal{Y}\left(Y_{W}\left(u, z_{0}\right) w, z_{2}\right),
\end{aligned}
$$

for all $u \in V$ and $w \in W$.

These axioms imply that the intertwining vertex operators satisfy translation, locality, associativity, commutativity and skew-symmetry:

$$
\begin{aligned}
& \mathcal{Y}\left(L_{W}(-1) w, z\right)=\partial_{z} \mathcal{Y}(w, z), \\
& \left(z_{1}-z_{2}\right)^{m} Y_{W}\left(u, z_{1}\right) \mathcal{Y}\left(w, z_{2}\right) \\
& =\left(z_{1}-z_{2}\right)^{m} \mathcal{Y}\left(w, z_{2}\right) Y\left(u, z_{1}\right), \\
& \left(z_{0}+z_{2}\right)^{n} Y_{W}\left(u, z_{0}+z_{2}\right) \mathcal{Y}\left(w, z_{2}\right) v \\
& =\left(z_{0}+z_{2}\right)^{n} \mathcal{Y}\left(Y_{W}\left(u, z_{0}\right) w, z_{2}\right) v, \\
& u_{W}(k) \mathcal{Y}(w, z)-\mathcal{Y}(w, z) u(k) \\
& =\sum_{j \geq 0}\left(\begin{array}{c}
k \\
j
\end{array}\right) \mathcal{Y}\left(u_{W}(j) w, z\right) z^{k-j}, \\
& \mathcal{Y}(w, z) v=\mathrm{e}^{z L_{W}(-1)} Y_{W}(v,-z) w,
\end{aligned}
$$

for $u, v \in V, w \in W$ and integers $m, n \gg 0$.

\subsection{Example: Heisenberg Intertwiners}

Consider the Heisenberg vertex operator algebra $M$,
[10] generated by weight one normalized Heisenberg vector $a$ with modes obeying

$$
[a(n), a(m)]=n \delta_{n,-m}, n, m \in \mathbb{Z} .
$$

In [3] we consider an extension $\mathcal{M}=\cup_{\alpha \in \mathbb{C}} M_{\alpha}$ of $M$ by its irreducible modules $M_{\alpha}$ generated by a $\mathbb{C}$-valued continuous parameter $\alpha$ automorphism $g=\mathrm{e}^{2 \pi i \alpha a(0)}$.

We introduce an extra operator $q$ which is canonically conjugate to the zero mode $a(0)$, i.e.,

$$
[a(n), q]=\delta_{n, 0} \text {. }
$$

The state $1 \otimes \mathrm{e}^{\alpha} \in \mathcal{M}$ is created by the action of $\mathrm{e}^{\alpha q}$ on the state $1 \otimes \mathrm{e}^{0}$. Using $q$-conjugation and associativity properties, we explicitly construct in [3] the creative intertwining operators $\mathcal{Y}(u, z): M \rightarrow M_{\alpha}$. We then prove:

Theorem 1 (Tuite-Z) The creative intertwining operators $\mathcal{Y}$ for $\mathcal{M}$ are generated by $q$-conjugation of vertex operators of $M$. For a Heisenberg state $u$,

$$
\begin{aligned}
& \mathcal{Y}\left(u \otimes \mathrm{e}^{\alpha}, z\right)=\mathrm{e}^{\alpha q} Y_{-}\left(\mathrm{e}^{\alpha}, z\right) Y\left(u \otimes \mathrm{e}^{0}\right) Y_{+}\left(\mathrm{e}^{\alpha}, z\right) z^{\alpha a(0)}, \\
& Y_{ \pm}\left(\mathrm{e}^{\alpha}, z\right) \equiv \exp \left(\mp \alpha \sum_{n>0} a( \pm n) \frac{z^{\mp n}}{n}\right) .
\end{aligned}
$$

The operators $\mathcal{Y}$ with some extra cocycle structure satisfy a natural extension from rational to complex parameters of the notion of a Generalized VOA as described by Dong and Lepowsky $[7,12]$. We then prove in [3].

Theorem 2 (Tuite-Z) $\mathcal{Y}\left(u \otimes \mathrm{e}^{\alpha}, z\right)$ satisfy the generalized Jacobi identity

$$
\begin{aligned}
& z_{0}^{-1}\left(\frac{z_{1}-z_{2}}{z_{0}}\right)^{-\alpha \beta} \delta\left(\frac{z_{1}-z_{2}}{z_{0}}\right) \mathcal{Y}\left(u \otimes \mathrm{e}^{\alpha}, z_{1}\right) \mathcal{Y}\left(v \otimes \mathrm{e}^{\beta}, z_{2}\right) \\
& -C(\alpha, \beta) z_{0}^{-1}\left(\frac{z_{2}-z_{1}}{z_{0}}\right)^{-\alpha \beta} \delta\left(\frac{z_{2}-z_{1}}{-z_{0}}\right) \mathcal{Y}\left(v \otimes \mathrm{e}^{\beta}, z_{2}\right) \mathcal{Y}\left(u \otimes \mathrm{e}^{\alpha}, z_{1}\right) \\
& =z_{2}^{-1} \delta\left(\frac{z_{1}-z_{0}}{z_{2}}\right) \mathcal{Y}\left(\mathcal{Y}\left(u \otimes \mathrm{e}^{\alpha}, z_{0}\right)\left(v \otimes \mathrm{e}^{\beta}\right), z_{2}\right)\left(\frac{z_{1}-z_{0}}{z_{2}}\right)^{\alpha a(0)},
\end{aligned}
$$

for all $u \otimes \mathrm{e}^{\alpha}, v \otimes \mathrm{e}^{\beta} \in \mathcal{M}$.

\subsection{Invariant Form for the Extended Heisenberg Algebra}

The definitions of invariant forms $[8,13]$ for a VOSA and its $g$-twisted modules were given by Scheithauer [14] and in [2] correspondingly. A bilinear form $\langle\cdot, \cdot\rangle$ on $\mathcal{M}$ is said to be invariant if for all $u \otimes \mathrm{e}^{\alpha}, v \otimes \mathrm{e}^{\beta}$, $w \otimes \mathrm{e}^{\gamma} \in \mathcal{M}$ we have

$$
\begin{aligned}
& \left\langle\mathcal{Y}\left(u \otimes \mathrm{e}^{\alpha}, z\right) v \otimes \mathrm{e}^{\beta}, w \otimes \mathrm{e}^{\gamma}\right\rangle=\mathrm{e}^{i \pi \alpha \beta}\left\langle v \otimes \mathrm{e}^{\beta}, \mathcal{Y}^{\dagger}\left(u \otimes \mathrm{e}^{\alpha}, z\right) w \otimes \mathrm{e}^{\gamma}\right\rangle, \\
& \mathcal{Y}^{\dagger}\left(u \otimes \mathrm{e}^{\alpha}, z\right)=\mathcal{Y}\left(\mathrm{e}^{-z \lambda^{-2} L(1)}\left(-\frac{\lambda}{z}\right)^{2 L(0)}\left(u \otimes \mathrm{e}^{\alpha}\right),-\frac{\lambda^{2}}{z}\right) .
\end{aligned}
$$


We are interested in the Möbius map $z \mapsto w=\frac{\rho}{z}$ associated with the sewing condition so that $\lambda=-\xi \rho^{\frac{1}{2}}$, with $\xi \in\{ \pm \sqrt{-1}\}$. We prove in [3]

Theorem 3 (Tuite-Z) The invariant form $\langle.,$.$\rangle on$ $\mathcal{M}$ is symmetric, unique and invertible with

$$
\left\langle v \otimes \mathrm{e}^{\alpha}, w \otimes \mathrm{e}^{\beta}\right\rangle=\lambda^{-\alpha^{2}} \delta_{\alpha,-\beta}\left\langle v \otimes \mathrm{e}^{0}, w \otimes \mathrm{e}^{0}\right\rangle .
$$

\subsection{Rank Two Free Fermionic Vertex Operator Super Algebra}

Consider the Vertex Operator Super Algebra (VOSA) generated by

$$
Y\left(\psi^{ \pm}, z\right)=\sum_{n \in \mathbb{Z}} \psi^{ \pm}(n) z^{-n-1}
$$

for two vectors $\psi^{ \pm}$with modes satisfying anti-commutation relations

$$
\begin{aligned}
& {\left[\psi^{+}(m), \psi^{-}(n)\right]_{+}=\delta_{m,-n-1},} \\
& {\left[\psi^{ \pm}(m), \psi^{ \pm}(n)\right]_{+}=0 .}
\end{aligned}
$$

The VOSA vector space $V=\oplus_{k \geq 0} V_{k / 2}$ is a Fock space with basis vectors

$$
\boldsymbol{\Psi}(\mathbf{k}, \mathbf{l}) \equiv \psi^{+}\left(-k_{1}\right) \cdots \psi^{+}\left(-k_{s}\right) \psi^{-}\left(-l_{1}\right) \cdots \psi^{-}\left(-l_{t}\right) \mathbf{1},
$$

of weight

$$
w t[\mathbf{\Psi}(\mathbf{k}, \mathbf{l})]=\sum_{i}\left(k_{i}+\frac{1}{2}\right)+\sum_{j}\left(l_{j}+\frac{1}{2}\right),
$$

where $1 \leq k_{1}<k_{2}<\cdots<k_{s}$ and $1 \leq l_{1}<l_{2}<\cdots<l_{t}$ with $\psi^{ \pm}(k) \mathbf{1}=0$ for all $k \geq 0$.

\subsection{Rank Two Fermionic Vertex Operator Super Algebra}

The conformal vector is

$$
\omega=\frac{1}{2}\left[\psi^{+}(-2) \psi^{-}(-1)+\psi^{-}(-2) \psi^{+}(-1)\right] \mathbf{1},
$$

whose modes generate a Virasoro algebra of central charge 1 . $\psi^{ \pm}$has $L(0)$-weight $\frac{1}{2}$. The weight 1 subspace of $V$ is $V_{1}=\mathbb{C} a$, for normalized Heisenberg bosonic vector $a=\psi^{+}(-1) \psi^{-}(-1) \mathbf{1}$, the conformal vector, and the Virasoro grading operator are

$$
\begin{aligned}
& {[a(m), a(n)]=m \delta_{m,-n},} \\
& \omega=\frac{1}{2} a(-1)^{2} \mathbf{1}, \\
& L(0)=\frac{a(0)^{2}}{2}+\sum_{n>0} a(-n) a(n) .
\end{aligned}
$$

\section{Sewing of Riemann Surfaces}

\subsection{Basic Notions}

For standard homology basis $a_{i}, b_{j}$ with $i=1, \cdots, g$ on a genus $g$ Riemann surface $[15,16]$ consider the normalized differential of the second kind which is a symmetric meromorphic form with $\oint_{a_{i}} \omega^{(g)}(z, \cdot)=0$, has the form

$$
\omega^{(g)}\left(z_{1}, z_{2}\right) \sim \frac{d z_{1} d z_{2}}{\left(z_{1}-z_{2}\right)^{2}} \quad \text { for local coordinates } z_{1} \sim z_{2} .
$$

A normalized basis of holomorphic 1-forms $v_{i}$, the period matrix $\Omega_{i j}^{(g)}$, and normalized differential of the third kind are given by

$$
\begin{aligned}
& v_{i}^{(g)}(z)=\oint_{b_{i}} \omega^{(g)}(z, \cdot), \quad \oint_{a_{i}} v_{j}^{(g)}=2 \pi i \delta_{i j}, \\
& \Omega_{i j}^{(g)}=\frac{1}{2 \pi i} \oint_{b_{i}} v_{j}^{(g)}, \quad \omega_{p_{2}-p_{1}}^{(g)}(z)=\int_{p_{1}}^{p_{2}} \omega^{(g)}(z, \cdot),
\end{aligned}
$$

where $\oint_{a_{i}} \omega_{p_{2}-p_{1}}^{(g)}=0, \quad \omega_{p_{2}-p_{1}}^{(g)}(z) \sim \frac{(-1)^{a}}{z-p_{a}} \mathrm{~d} z$ for $z \sim p_{a}$, $a=1,2$.

\subsection{Period Matrix}

$\Omega^{(g)}$ is symmetric with positive imaginary part i.e. $\Omega^{(g)} \in \mathbb{H}_{g}$, the Siegel upper half plane. The canonical intersection form on cycles is preserved under the action of the symplectic group $S p(2 g, \mathbb{Z})$ where

$$
\left(\begin{array}{l}
b \\
a
\end{array}\right) \rightarrow\left(\begin{array}{l}
\tilde{b} \\
\tilde{a}
\end{array}\right)=\left(\begin{array}{ll}
A & B \\
C & D
\end{array}\right)\left(\begin{array}{l}
b \\
a
\end{array}\right), \quad\left(\begin{array}{ll}
A & B \\
C & D
\end{array}\right) \in S p(2 g, \mathbb{Z}) .
$$

This induces the modular action on $\mathbb{H}_{g}$

$$
\Omega^{(g)} \rightarrow \tilde{\Omega}^{(g)}=\left(A \Omega^{(g)}+B\right)\left(C \Omega^{(g)}+D\right)^{-1} .
$$

\subsection{Sewing Two Tori to Form a Genus Two Riemann Surface}

Consider $[1,17]$ two oriented tori $\Sigma_{a}^{(1)}=\mathbb{C} / \Lambda_{\tau_{a}}$ with $a=1,2$ for $\Lambda_{\tau_{a}}=2 \pi i\left(\mathbb{Z} \oplus \tau_{a} \mathbb{Z}\right)$ for $\tau_{a} \in \mathbb{H}_{1}$, the complex upper half plane. For $z_{a} \in \Sigma_{a}^{(1)}$ the closed disk $\left|z_{a}\right| \leq r_{a}$ is contained in $\Sigma_{a}^{(1)}$ provided $r_{a}<\frac{1}{2} D\left(\tau_{a}\right)$ where

$$
D\left(\tau_{a}\right)=\min _{\lambda \in \Lambda_{\tau_{a}}, \lambda \neq 0}|\lambda|=\text { min imal lattice distance. }
$$

Introduce a sewing parameter $\varepsilon \in \mathbb{C}$ and excise the disks $\left|z_{1}\right| \leq|\varepsilon| / r_{2}$ and $\left|z_{2}\right| \leq|\varepsilon| / r_{1}$ where

$$
|\varepsilon| \leq r_{1} r_{2}<\frac{1}{4} D\left(\tau_{1}\right) D\left(\tau_{2}\right) \text {. }
$$


Identify the annular regions $|\varepsilon| / r_{2} \leq\left|z_{1}\right| \leq r_{1}$ and $|\varepsilon| / r_{1} \leq\left|z_{2}\right| \leq r_{2}$ via the sewing relation

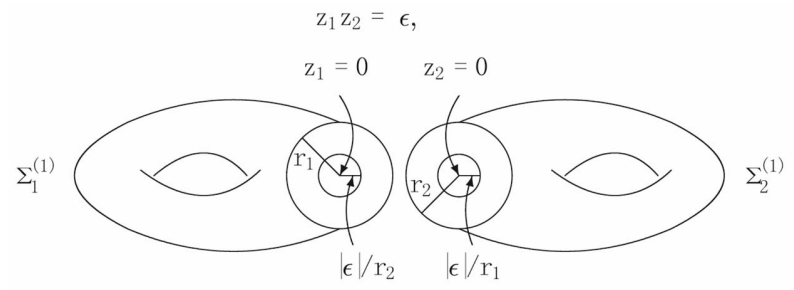

gives a genus two Riemann surface $\Sigma^{(2)}$ parameterized by the domain

$$
\mathcal{D}^{\varepsilon}=\left\{\left(\tau_{1}, \tau_{2}, \varepsilon\right) \in \mathbb{H}_{1} \times \mathbb{H}_{1} \times \mathbb{C}|| \varepsilon \mid<\frac{1}{4} D\left(\tau_{1}\right) D\left(\tau_{2}\right)\right\} .
$$

\subsection{Torus Self-Sewing to Form a Genus Two Riemann Surface}

In [1] we describe procedures of sewing Riemann surfaces [17]. Consider a self-sewing of the oriented torus $\Sigma^{(1)}=\mathbb{C} / \Lambda, \quad \Lambda=2 \pi i(\mathbb{Z} \tau \oplus \mathbb{Z}), \quad \tau \in \mathbb{H}_{1}$.

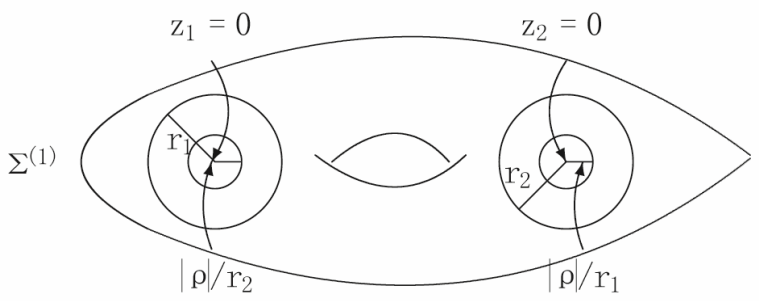

Define the annuli $\mathcal{A}_{a}, a=1,2$ centered at $\mathrm{Z}=0$ and $Z=W$ of $\Sigma^{(1)}$ with local coordinates $Z_{1}=Z$ and $z_{2}=z-w$ respectively. We use the convention $\overline{1}=2$, $\overline{2}=1$. Take the outer radius of $\mathcal{A}_{a}$ to be

$$
r_{a}<\frac{1}{2} D(q)=\min _{\lambda \in \Lambda, \lambda \neq 0}|\lambda| \text {. }
$$

Introduce a complex parameter $\rho,|\rho| \leq r_{1} r_{2}$. Take inner radius to be $|\rho| / r_{\bar{a}}$, with $|\rho| \leq r_{1} r_{2} . r_{1}, r_{2}$ must be sufficiently small to ensure that the disks do not intersect. Excise the disks

$$
\left\{z_{a},\left|z_{a}\right|<|\rho| r_{\bar{a}}^{-1}\right\} \subset \Sigma^{(1)},
$$

to form a twice-punctured surface

$$
\hat{\Sigma}^{(1)}=\Sigma^{(1)} \backslash \bigcup_{a=1,2}\left\{z_{a},\left|z_{a}\right|<|\rho| r_{\bar{a}}^{-1}\right\} .
$$

Identify the annular regions $\mathcal{A}_{a} \subset \hat{\Sigma}^{(1)}$,

$$
\mathcal{A}_{a}=\left\{z_{a},|\rho| r_{\bar{a}}^{-1} \leq\left|z_{a}\right| \leq r_{a}\right\}
$$

as a single region $\mathcal{A}=\mathcal{A}_{1} \simeq \mathcal{A}_{2}$ via the sewing relation

$$
z_{1} z_{2}=\rho,
$$

to form a compact genus two Riemann surface

$$
\Sigma^{(2)}=\hat{\Sigma}^{(1)} \backslash\left\{\mathcal{A}_{1} \cup \mathcal{A}_{2}\right\} \cup \mathcal{A},
$$

parameterized by

$$
\begin{aligned}
& \mathcal{D}^{\rho}= \\
& \left\{(\tau, w, \rho) \in \mathbb{H}_{1} \times \mathbb{C} \times \mathbb{C},|w-\lambda|>2|\rho|^{\frac{1}{2}}>0, \lambda \in \Lambda\right\} .
\end{aligned}
$$

\section{Elliptic Functions}

\subsection{Weierstrass Function}

The Weierstrass $\wp$-function periodic in $z$ with periods $2 \pi i$ and $2 \pi i \tau$ is

$$
\begin{aligned}
\wp(z, \tau) & =\frac{1}{z^{2}}+\sum_{\substack{m, n \in \mathbb{Z} \\
(m, n) \neq(0,0)}}\left[\frac{1}{\left(z-\omega_{m, n}\right)^{2}}-\frac{1}{\omega_{m, n}^{2}}\right] \\
& =\frac{1}{z^{2}}+\sum_{n \geq 4, n \text { even }}(n-1) E_{n}(\tau) z^{n-2},
\end{aligned}
$$

for $(z, \tau) \in \mathbb{C} \times \mathbb{H}, \omega_{m, n}=2 \pi i(m \tau+n)$. We define for $k \geq 1$,

$$
\begin{aligned}
P_{k}(z, \tau) & =\frac{(-1)^{k-1}}{(k-1) !} \frac{\partial^{k-1}}{\partial z^{k-1}} P_{1}(z, \tau) \\
& =\frac{1}{z^{k}}+(-1)^{k} \sum_{n \geq k}\left(\begin{array}{c}
n-1 \\
k-1
\end{array}\right) E_{n}(\tau) z^{n-k} .
\end{aligned}
$$

Then

$$
P_{2}(z, \tau)=\wp(z, \tau)+E_{2}(\tau) .
$$

$P_{k}$ has periodicities

$$
\begin{aligned}
& P_{k}(z+2 \pi i, \tau)=P_{k}(z, \tau), \\
& P_{k}(z+2 \pi i \tau, \tau)=P_{k}(z, \tau)-\delta_{k 1} .
\end{aligned}
$$

\subsection{Eisenstein Series}

The Eisenstein series $E_{n}(\tau)$ is equal to 0 for $n$ odd, and for $n$

$$
E_{n}(\tau)=-\frac{B_{n}(0)}{n !}+\frac{2}{(n-1) !} \sum_{r \geq 1} \frac{r^{n-1} q^{r}}{1-q^{r}},
$$

where $B_{n}(0)$ is the $n$th Bernoulli number. If $n \geq 4$ then $E_{n}(\tau)$ is a holomorphic modular form of weight $n$ on $S L(2, \mathbb{Z})$

$$
E_{n}(\gamma \cdot \tau)=(c \tau+d)^{n} E_{n}(\tau)
$$

for all $\gamma=\left(\begin{array}{ll}a & b \\ c & d\end{array}\right) \in S L(2, \mathbb{Z})$, where $\gamma \cdot \tau=\frac{a \tau+b}{c \tau+d}$. 
$E_{2}(\tau)$ is a quasimodular form

$$
E_{2}(\gamma \cdot \tau)=(c \tau+d)^{2} E_{2}(\tau)-\frac{c(c \tau+d)}{2 \pi i},
$$

having the exceptional transformation law.

\subsection{The Theta Function}

We recall the definition of the theta function with real characteristics [18]

$$
\begin{aligned}
& \vartheta^{(g)}\left[\begin{array}{l}
\alpha \\
\beta
\end{array}\right]\left(z \mid \Omega^{(g)}\right)= \\
& \sum_{m \in \mathbb{Z}^{g}} \exp \left(i \pi(m+\alpha) \cdot \Omega^{(g)} \cdot(m+\alpha)+(m+\alpha) \cdot(z+2 \pi i \beta)\right),
\end{aligned}
$$

for

$$
\begin{aligned}
& \alpha=\left(\alpha_{j}\right), \beta=\left(\beta_{j}\right) \in \mathbb{R}^{g}, z=\left(z_{j}\right) \in \mathbb{C}^{g}, \\
& \theta_{j}=-\mathrm{e}^{-2 \pi i \beta_{j}}, \phi_{j}=-\mathrm{e}^{2 \pi i \alpha_{j}}, j=1, \cdots, g, \\
& \vartheta^{(g)}\left[\begin{array}{c}
\alpha \\
\beta
\end{array}\right]\left(z+2 \pi i\left(\Omega^{(g)} \cdot r+s\right) \mid \Omega^{(g)}\right) \\
& =\mathrm{e}^{2 \pi i \alpha \cdot s} \mathrm{e}^{-2 \pi i \beta \cdot r} \mathrm{e}^{-i \pi r \cdot \Omega^{(g)} \cdot r-r \cdot z} \vartheta^{(g)}\left[\begin{array}{c}
\alpha \\
\beta
\end{array}\right]\left(z \mid \Omega^{(g)}\right), \\
& \vartheta^{(g)}\left[\begin{array}{c}
\alpha+r \\
\beta+s
\end{array}\right]\left(z \mid \Omega^{(g)}\right)=\mathrm{e}^{2 \pi i \alpha \cdot s} \vartheta^{(g)}\left[\begin{array}{l}
\alpha \\
\beta
\end{array}\right]\left(z \mid \Omega^{(g)}\right),
\end{aligned}
$$

for $r, s \in \mathbb{Z}^{g}$.

\subsection{Twisted Elliptic Functions}

Let $(\theta, \phi) \in U(1) \times U(1)$ denote a pair of modulus one complex parameters with $\phi=\exp (2 \pi i \lambda)$ for $0 \leq \lambda<1$. For $z \in \mathbb{C}$ and $\tau \in \mathbb{H}$ we define "twisted" Weierstrass functions for $k \geq 1 \quad[19,20]$

$$
P_{k}\left[\begin{array}{l}
\theta \\
\phi
\end{array}\right](z, \tau)=\frac{(-1)^{k}}{(k-1) !} \sum_{n \in \mathbb{Z}+\lambda}^{\prime} \frac{n^{k-1} q_{z}^{n}}{1-\theta^{-1} q^{n}},
$$

for $q=q_{2 \pi i \tau}$ where $\sum$ means we omit $n=0$ if

$$
(\theta, \phi)=(1,1) . \quad P_{k}\left[\begin{array}{l}
\theta \\
\phi
\end{array}\right]
$$

converges absolutely and uniformly on compact subsets of the domain $|q|<\left|q_{z}\right|<1 \quad$ [20].

Lemma 1 (Mason-Tuite-Z) For $(\theta, \phi) \neq(1,1)$,

$$
P_{k}\left[\begin{array}{l}
\theta \\
\phi
\end{array}\right](z, \tau)
$$

is periodic in $\mathrm{z}$ with periods $2 \pi i \tau$ and $2 \pi i$ with multipliers $\theta$ and $\phi$ respectively.

\subsection{Modular Properties of Twisted Weierstrass Functions}

Define the standard left action of the modular group for $\gamma=\left(\begin{array}{ll}a & b \\ c & d\end{array}\right) \in \Gamma=S L(2, \mathbb{Z})$ on $(z, \tau) \in \mathbb{C} \times \mathbb{H}$ with

$$
\gamma \cdot(z, \tau)=(\gamma \cdot z, \gamma \cdot \tau)=\left(\frac{z}{c \tau+d}, \frac{a \tau+b}{c \tau+d}\right) .
$$

We also define a left action of $\Gamma$ on $(\theta, \phi)$

$$
\gamma \cdot\left[\begin{array}{l}
\theta \\
\phi
\end{array}\right]=\left[\begin{array}{l}
\theta^{a} \phi^{b} \\
\theta^{c} \phi^{d}
\end{array}\right] .
$$

Then we obtain:

Theorem 4 (Mason-Tuite-Z) For $(\theta, \phi) \neq(1,1)$ we have

$$
P_{k}\left(\gamma \cdot\left[\begin{array}{l}
\theta \\
\phi
\end{array}\right]\right)(\gamma \cdot z, \gamma \cdot \tau)=(c \tau+d)^{k} P_{k}\left[\begin{array}{l}
\theta \\
\phi
\end{array}\right](z, \tau) .
$$

\subsection{Twisted Eisenstein Series}

We introduce twisted Eisenstein series for $n \geq 1$,

$$
\begin{aligned}
E_{n}\left[\begin{array}{l}
\theta \\
\phi
\end{array}\right](\tau)= & -\frac{B_{n}(\lambda)}{n !}+\frac{1}{(n-1) !} \sum_{r \geq 0}^{\prime} \frac{(r+\lambda)^{n-1} \theta^{-1} q^{r+\lambda}}{1-\theta^{-1} q^{r+\lambda}} \\
& +\frac{(-1)^{n}}{(n-1) !} \sum_{r \geq 1} \frac{(r-\lambda)^{n-1} \theta q^{r-\lambda}}{1-\theta q^{r-\lambda}},
\end{aligned}
$$

where $\sum^{\prime}$ means we omit $r=0$ if $(\theta, \phi)=(1,1)$ and where $B_{n}(\lambda)$ is the Bernoulli polynomial defined by

$$
\frac{q_{z}^{\lambda}}{q_{z}-1}=\frac{1}{z}+\sum_{n \geq 1} \frac{B_{n}(\lambda)}{n !} z^{n-1}
$$

In particular

$$
B_{1}(\lambda)=\lambda-\frac{1}{2}
$$

Note that

$$
E_{n}\left[\begin{array}{l}
1 \\
1
\end{array}\right](\tau)=E_{n}(\tau),
$$

the standard Eisenstein series for even $n \geq 2$, whereas

$$
E_{n}\left[\begin{array}{l}
1 \\
1
\end{array}\right](\tau)=-B_{1}(0) \delta_{n, 1}=\frac{1}{2} \delta_{n, 1}
$$

for $n$ odd.

Theorem 5 (Mason-Tuite-Z) We have

$$
P_{k}\left[\begin{array}{l}
\theta \\
\phi
\end{array}\right](z, \tau)=\frac{1}{z^{k}}+(-1)^{k} \sum_{n \geq k}\left(\begin{array}{l}
n-1 \\
k-1
\end{array}\right) E_{n}\left[\begin{array}{l}
\theta \\
\phi
\end{array}\right](\tau) z^{n-k} .
$$


Theorem 6 (Mason-Tuite-Z) For $(\theta, \phi) \neq(1,1)$, $E_{k}\left[\begin{array}{l}\theta \\ \phi\end{array}\right]$ is a modular form of weight $k$ where

$$
E_{k}\left(\gamma \cdot\left[\begin{array}{l}
\theta \\
\phi
\end{array}\right]\right)(\gamma \cdot \tau)=(c \tau+d)^{k} E_{k}\left[\begin{array}{l}
\theta \\
\phi
\end{array}\right](\tau)
$$

\subsection{Twisted Elliptic Functions}

In particular,

$$
\begin{aligned}
& P_{1}\left[\begin{array}{l}
\theta \\
\phi
\end{array}\right](z, \tau)=\frac{1}{z}-\sum_{k \geq 1} E_{k}\left[\begin{array}{l}
\theta \\
\phi
\end{array}\right](\tau) z^{k-1}, \\
& E_{k}\left[\begin{array}{l}
\theta \\
\phi
\end{array}\right](\tau)=\frac{1}{(2 \pi i)^{k}} \sum_{m}\left[\sum_{n}^{\prime} \frac{\theta^{m} \phi^{n}}{(m \tau+n)^{k}}\right] . \\
& P_{1}\left[\begin{array}{l}
\theta \\
\phi
\end{array}\right]\left(z-z^{\prime}, \tau\right)=\frac{1}{z-z^{\prime}}+\sum_{k, l \geq 1} C\left[\begin{array}{l}
\theta \\
\phi
\end{array}\right](k, l) z^{k-1} z^{\prime l-1},
\end{aligned}
$$

where

$$
\begin{aligned}
& C\left[\begin{array}{l}
\theta \\
\phi
\end{array}\right](k, l, \tau)=(-1)^{l}\left(\begin{array}{c}
k+l-2 \\
k-1
\end{array}\right) E_{k+l-1}\left[\begin{array}{l}
\theta \\
\phi
\end{array}\right](\tau), \\
& P_{1}\left[\begin{array}{l}
\theta \\
\phi
\end{array}\right]\left(w+z-z^{\prime}, \tau\right)=\sum_{k, l \geq 1} D\left[\begin{array}{l}
\theta \\
\phi
\end{array}\right](k, l, w) z^{k-1} z^{\prime l-1},
\end{aligned}
$$

and

$$
\begin{aligned}
& D\left[\begin{array}{l}
\theta \\
\phi
\end{array}\right](k, l, \tau, z) \\
& =(-1)^{k+1}\left(\begin{array}{c}
k+l-2 \\
k-1
\end{array}\right) P_{k+l-1}\left[\begin{array}{l}
\theta \\
\phi
\end{array}\right](\tau, z) .
\end{aligned}
$$

\section{The Prime Form}

There exists a (nonsingular and odd) character $\left[\begin{array}{l}\gamma \\ \delta\end{array}\right]$ such that $[18,21,22]$

$$
\begin{gathered}
\vartheta\left[\begin{array}{l}
\gamma \\
\delta
\end{array}\right]\left(0 \mid \Omega^{(g)}\right)=0, \\
\partial_{z_{i}} \vartheta\left[\begin{array}{l}
\gamma \\
\delta
\end{array}\right]\left(0 \mid \Omega^{(g)}\right) \neq 0 .
\end{gathered}
$$

Let

$$
\zeta(z)=\sum_{i=1}^{g} \partial_{z_{i}} \vartheta\left[\begin{array}{l}
\gamma \\
\delta
\end{array}\right]\left(0 \mid \Omega^{(g)}\right) v_{i}^{(g)}(z),
$$

be a holomorphic 1-form, and let $\zeta(z)^{\frac{1}{2}}$ denote the form of weight $\frac{1}{2}$ on the double cover $\tilde{\Sigma}^{(g)}$ of $\Sigma^{(g)}$.
We define the prime form

$$
\begin{gathered}
E^{(g)}\left(z, z^{\prime}\right)=\frac{\vartheta\left[\begin{array}{l}
\gamma \\
\delta
\end{array}\right]\left(\int_{z^{\prime}}^{z} v^{(g)} \mid \Omega^{(g)}\right)}{\zeta(z)^{\frac{1}{2}} \zeta\left(z^{\prime}\right)^{\frac{1}{2}}} \\
\sim\left(z-z^{\prime}\right) \mathrm{d} z^{-\frac{1}{2}} \mathrm{~d}^{\prime}{ }^{-\frac{1}{2}} \quad \text { for } z \sim z^{\prime} .
\end{gathered}
$$

The prime form is anti-symmetic,

$$
E^{(g)}\left(z, z^{\prime}\right)=-E^{(g)}\left(z^{\prime}, z\right),
$$

and a holomorphic differential form of weight

$$
\left(-\frac{1}{2},-\frac{1}{2}\right) \text { on } \tilde{\Sigma} \times \tilde{\Sigma} \text {, }
$$

and has multipliers 1 and $\mathrm{e}^{-i \pi \Omega_{j j}^{(g)}-\int_{z^{\prime}}^{Z} \gamma_{j}^{(g)}}$ along the $a_{i}$ and $b_{j}$ cycles in $z$ [21]. The normalized differentials of the second and third kind can be expressed in terms of the prime form [18]

$$
\begin{aligned}
& \omega^{(g)}\left(z, z^{\prime}\right)=\partial_{z} \partial_{z^{\prime}} \log E^{(g)}\left(z, z^{\prime}\right) \mathrm{d} z d z^{\prime}, \\
& \omega_{p-q}^{(g)}(z)=\partial_{z} \log \frac{E^{(g)}(z, p)}{E^{(g)}(z, q)} \mathrm{d} z .
\end{aligned}
$$

Conversely, we can also express the prime form in terms of $\omega_{p-q}^{(g)}$ by [22]

$$
\begin{aligned}
& E^{(g)}\left(z, z^{\prime}\right)= \\
& \lim _{p \rightarrow z, q \rightarrow y}\left[\sqrt{(z-p)\left(q-z^{\prime}\right)} \exp \left(-\frac{1}{2} \int_{z^{\prime}}^{z} \omega_{p-q}^{(g)}\right)\right] \mathrm{d} z^{-\frac{1}{2}} \mathrm{~d} z^{\prime-\frac{1}{2}} .
\end{aligned}
$$

\section{Torus Prime Form}

The prime form on torus [18]

$$
\begin{aligned}
& E^{(1)}\left(z, z^{\prime}\right)=K^{(1)}\left(z-z^{\prime}, \tau\right) \mathrm{d} z^{-\frac{1}{2}} \mathrm{~d}^{\prime}{ }^{-\frac{1}{2}}, \\
& K^{(1)}(z, \tau)=\frac{\vartheta_{1}(z, \tau)}{\partial_{z} \vartheta_{1}(0, \tau)},
\end{aligned}
$$

for $z \in \mathbb{C}$ and $\tau \in \mathbb{H}_{1}$ and where

We have

$$
\vartheta_{1}(z, \tau)=\vartheta\left[\begin{array}{l}
\frac{1}{2} \\
\frac{1}{2}
\end{array}\right](z, \tau) \text {. }
$$

$$
\begin{aligned}
& K^{(1)}(z, \tau)=\exp \left(-P_{0}(z, \tau)\right), \\
& P_{0}(z, \tau)=-\log (z)+\sum_{k \geq 2} \frac{1}{k} E_{k}(\tau) z^{k},
\end{aligned}
$$




$$
P_{1}(z, \tau)=-\frac{\mathrm{d}}{\mathrm{d} z} P_{0}(z, \tau)=\frac{1}{z}-\sum_{k \geq 2} E_{k}(\tau) z^{k-1} .
$$

$K^{(1)}(z, \tau)$ has periodicities

$$
\begin{aligned}
& K^{(1)}(z+2 \pi i, \tau)=-K^{(1)}(z, \tau), \\
& K^{(1)}(z+2 \pi i \tau, \tau)=-q_{z}^{-1} q^{-1 / 2} K^{(1)}(z, \tau) .
\end{aligned}
$$

\section{The Szegö Kernel}

The Szegö Kernel $[18,21,22]$ is defined by

$$
\begin{aligned}
& S^{(g)}\left[\begin{array}{l}
\theta \\
\phi
\end{array}\right]\left(z, z^{\prime} \mid \Omega^{(g)}\right) \\
& =\frac{\vartheta\left[\begin{array}{l}
\alpha \\
\beta
\end{array}\right]\left(\int_{z^{\prime}}^{z} v^{(g)}\right)}{\vartheta\left[\begin{array}{l}
\alpha \\
\beta
\end{array}\right](0) E^{(g)}\left(z, z^{\prime}\right)} \sim \frac{\mathrm{dz}^{\frac{1}{2}} \mathrm{dz}^{\prime \frac{1}{2}}}{z-z^{\prime}} \text { for } z \sim z^{\prime},
\end{aligned}
$$

with $\vartheta\left[\begin{array}{l}\alpha \\ \beta\end{array}\right](0) \neq 0, \theta_{j}=-\mathrm{e}^{-2 \pi i \beta_{j}}, \phi_{j}=-\mathrm{e}^{2 \pi i \alpha_{j}}$,

$j=1, \cdots, g$, where $E^{(g)}\left(z_{1}, z_{2}\right)$ is the genus $g$ prime form. The Szegö kernel has multipliers along the $a_{i}$ and $b_{j}$ cycles in $z$ given by $-\phi_{i}$ and $-\theta_{j}$ respectively and is a meromorphic $\left(\frac{1}{2}, \frac{1}{2}\right)$-form on $\tilde{\Sigma}^{(g)} \times \tilde{\Sigma}^{(g)}$

$$
S^{(g)}\left[\begin{array}{l}
\theta \\
\phi
\end{array}\right]\left(z, z^{\prime}\right)=-S^{(g)}\left[\begin{array}{l}
\theta^{-1} \\
\phi^{-1}
\end{array}\right]\left(z, z^{\prime}\right)
$$

where $\theta^{-1}=\left(\theta_{i}^{-1}\right)$ and $\phi^{-1}=\left(\phi_{i}^{-1}\right)$.

Finally, we describe the modular invariance of the Szegö kernel under the symplectic group $S p(2 g, \mathbb{Z})$ where we find [21]

$$
S^{(g)}\left[\begin{array}{c}
\tilde{\theta} \\
\tilde{\phi}
\end{array}\right]\left(z, z^{\prime} \mid \tilde{\Omega}^{(g)}\right)=S^{(g)}\left[\begin{array}{l}
\theta \\
\phi
\end{array}\right]\left(z, z^{\prime} \mid \Omega^{(g)}\right),
$$

with $\tilde{\theta}_{j}=-\mathrm{e}^{-2 \pi i \tilde{\beta}_{j}}, \tilde{\phi}_{j}=-\mathrm{e}^{2 \pi i \tilde{\alpha}_{j}}$,

$$
\begin{aligned}
& \left(\begin{array}{c}
-\tilde{\beta} \\
\tilde{\alpha}
\end{array}\right)=\left(\begin{array}{ll}
A & B \\
C & D
\end{array}\right)\left(\begin{array}{c}
-\beta \\
\alpha
\end{array}\right)+\frac{1}{2}\left(\begin{array}{c}
-\operatorname{diag}\left(A B^{T}\right) \\
\operatorname{diag}\left(C D^{T}\right)
\end{array}\right), \\
& \tilde{\Omega}^{(g)}=\left(A \Omega^{(g)}+B\right)\left(C \Omega^{(g)}+D\right)^{-1},
\end{aligned}
$$

where $\operatorname{diag}(M)$ denotes the diagonal elements of a matrix $M$.

\subsection{Modular Properties of the Szegö Kernel}

Finally, we describe the modular invariance of the Szegö kernel under the symplectic group $S p(2 g, \mathbb{Z})$ where we find [21]

$$
S^{(g)}\left[\begin{array}{c}
\tilde{\theta} \\
\tilde{\phi}
\end{array}\right]\left(z, z^{\prime} \mid \tilde{\Omega}^{(g)}\right)=S^{(g)}\left[\begin{array}{l}
\theta \\
\phi
\end{array}\right]\left(z, z^{\prime} \mid \Omega^{(g)}\right),
$$

where $\tilde{\theta}_{j}=-\mathrm{e}^{-2 \pi i \tilde{\beta}_{j}}, \tilde{\phi}_{j}=-\mathrm{e}^{2 \pi i \tilde{\alpha}_{j}}$ for

$$
\left(\begin{array}{c}
-\tilde{\beta} \\
\tilde{\alpha}
\end{array}\right)=\left(\begin{array}{cc}
A & B \\
C & D
\end{array}\right)\left(\begin{array}{c}
-\beta \\
\alpha
\end{array}\right)+\frac{1}{2}\left(\begin{array}{c}
-\operatorname{diag}\left(A B^{T}\right) \\
\operatorname{diag}\left(C D^{T}\right)
\end{array}\right),
$$

where $\operatorname{diag}(M)$ denotes the diagonal elements of a matrix $M$.

\subsection{Torus Szegö Kernel}

On the torus $\Sigma^{(1)}$ the Szegö kernel for $(\theta, \phi) \neq(1,1)$ is

$$
S^{(1)}\left[\begin{array}{l}
\theta \\
\phi
\end{array}\right]\left(z, z^{\prime} \mid \tau\right)=P_{1}\left[\begin{array}{l}
\theta \\
\phi
\end{array}\right]\left(z-z^{\prime}, \tau\right) \mathrm{d} z^{\frac{1}{2}} \mathrm{~d}^{\prime \frac{1}{2}},
$$

where

$$
\begin{aligned}
P_{1}\left[\begin{array}{l}
\theta \\
\phi
\end{array}\right](z, \tau) & =\frac{\vartheta\left[\begin{array}{l}
\alpha \\
\beta
\end{array}\right](z, \tau)}{\vartheta\left[\begin{array}{l}
\alpha \\
\beta
\end{array}\right](0, \tau)} \frac{\partial_{z} \vartheta_{1}(0, \tau)}{\vartheta_{1}(z, \tau)} \\
& =-\sum_{k \in \mathbb{Z}} \frac{q_{z}^{k+\lambda}}{1-\theta^{-1} q^{k+\lambda}},
\end{aligned}
$$

for

$$
\vartheta_{1}(z, \tau)=\vartheta\left[\begin{array}{l}
\frac{1}{2} \\
\frac{1}{2}
\end{array}\right](z, \tau), \quad q_{z}=\mathrm{e}^{z}
$$

and

$$
\phi=\exp (2 \pi i \lambda) \text { for } 0 \leq \lambda<1 .
$$

\section{Structures on $\Sigma^{(2)}$ Constructed from Genus One Data}

Yamada (1980) described how to compute the period matrix and other structures on a genus $g$ Riemann surface in terms of lower genus data.

\section{1. $\omega^{(2)}$ on the Sewn Surface $\Sigma^{(2)}$}

$\omega^{(2)}$ can be determined from $\omega^{(1)}$ on each torus in Yamada's sewing scheme [17,23]. For a torus $\Sigma^{(1)}=\mathbb{C} / \Lambda_{\tau}$ the differential is

$$
\begin{aligned}
& \omega^{(1)}\left(z_{1}, z_{2}\right)=P_{2}\left(z_{1}-z_{2}, \tau\right) \mathrm{d} z_{1} \mathrm{~d} z_{2}, \\
& P_{2}(z, \tau)=\wp(z, \tau)+E_{2}(\tau),
\end{aligned}
$$

for Weierstrass function

$$
\wp(z, \tau)=\frac{1}{z^{2}}+\sum_{k \geq 4}(k-1) E_{k}(\tau) z^{k-2},
$$


and Eisenstein series for $k \geq 2$

$$
E_{k}(\tau)=\frac{1}{(2 \pi i)^{k}} \sum_{m}\left[\sum_{n}^{\prime} \frac{1}{(m \tau+n)^{k}}\right] .
$$

$E_{k}$ vanishes for odd $k$ and is a weight $k$ modular form for $k \geq 4 . E_{2}$ is a quasi-modular form. Expanding

$$
\begin{aligned}
& P_{2}\left(z_{1}-z_{2}, \tau\right)=\frac{1}{\left(z_{1}-z_{2}\right)^{2}}+\sum_{k, l \geq 1} C(k, l) z_{1}^{k-1} z_{2}^{l-1}, \\
& C(k, l)=C(k, l, \tau)=(-1)^{k+1} \frac{(k+l-1) !}{(k-1) !(l-1) !} E_{k+l}(\tau),
\end{aligned}
$$

we compute $\omega^{(2)}\left(z_{1}, z_{2}\right)$ in the sewing scheme in terms of the following genus one data, $a=1,2$

$$
A_{a}\left(k, l, \tau_{a}, \varepsilon\right)=\frac{\varepsilon^{(k+l) / 2}}{\sqrt{k l}} C\left(k, l, \tau_{a}\right) .
$$

\subsection{A Determinant and the Period Matrix}

Consider the infinite matrix $I-A_{1} A_{2}$ where $I$ is the infinite identity matrix and $\operatorname{define} \operatorname{det}\left(I-A_{1} A_{2}\right)$ by

$$
\begin{aligned}
\operatorname{logdet}\left(I-A_{1} A_{2}\right) & =\operatorname{Tr} \log \left(I-A_{1} A_{2}\right) \\
& =-\sum_{n \geq 1} \frac{1}{n} \operatorname{Tr}\left(\left(A_{1} A_{2}\right)^{n}\right),
\end{aligned}
$$

as a formal power series in $\varepsilon$ [23].

Theorem 7 (Mason-Tuite)

a) The infinite matrix

$$
\left(I-A_{1} A_{2}\right)^{-1}=\sum_{n \geq 0}\left(A_{1} A_{2}\right)^{n},
$$

is convergent for $\left(\tau_{1}, \tau_{2}, \varepsilon\right) \in \mathcal{D}^{\varepsilon}$.

b) $\operatorname{det}\left(I-A_{1} A_{2}\right)$ is non-vanishing and holomorphic on $\mathcal{D}^{\varepsilon}$

Furthermore we may obtain an explicit formula for the genus two period matrix $\Omega=\Omega^{(2)}$ on $\Sigma^{(2)}$ [23].

Theorem 8 (Mason-Tuite) $\Omega=\Omega\left(\tau_{1}, \tau_{2}, \varepsilon\right)$ is holomorphic on $\mathcal{D}^{\varepsilon}$ and is given by

$$
\begin{aligned}
& 2 \pi i \Omega_{11}=2 \pi i \tau_{1}+\varepsilon\left(A_{2}\left(I-A_{1} A_{2}\right)^{-1}\right)(1,1), \\
& 2 \pi i \Omega_{22}=2 \pi i \tau_{2}+\varepsilon\left(A_{1}\left(I-A_{2} A_{1}\right)^{-1}\right)(1,1),
\end{aligned}
$$

$$
S_{\kappa}^{(1)}\left[\begin{array}{l}
\theta_{1} \\
\phi_{1}
\end{array}\right](x, y \mid \tau, w)=\left(\frac{\vartheta_{1}(x-w, \tau) \vartheta_{1}(y, \tau)}{\vartheta_{1}(x, \tau) \vartheta_{1}(y-w, \tau)}\right)^{\kappa} \cdot \frac{\vartheta^{(1)}\left[\begin{array}{l}
\alpha_{1} \\
\beta_{1}
\end{array}\right](x-y+\kappa w, \tau)}{\vartheta^{(1)}\left[\begin{array}{c}
\alpha_{1} \\
\beta_{1}
\end{array}\right](\kappa w, \tau) K^{(1)}(x-y, \tau)} d x^{\frac{1}{2}} \mathrm{~d} y^{\frac{1}{2}}
$$

with similar expression for $S_{-\frac{1}{2}}^{(1)}(x, y)$ for $\kappa=-\frac{1}{2}$.

$$
2 \pi i \Omega_{12}=-\varepsilon\left(I-A_{1} A_{2}\right)^{-1}(1,1) .
$$

Here $(1,1)$ refers to the $(1,1)$-entry of a matrix.

\subsection{Genus Two Szegö Kernel on $\Sigma^{(2)}$ in the $\varepsilon$-Formalism}

We may compute $S^{(2)}\left[\begin{array}{l}\theta \\ \phi\end{array}\right]\left(z, z^{\prime}\right)$ for $\theta=\left(\theta_{1}, \theta_{2}\right)$ in the sewing scheme in terms of the genus one data

$$
\begin{aligned}
F_{a}(k, l) & =F_{a}\left[\begin{array}{l}
\theta_{a} \\
\phi_{a}
\end{array}\right]\left(k, l, \tau_{a}, \varepsilon\right) \\
& =\varepsilon^{\frac{1}{2}(k+l-1)} C\left[\begin{array}{l}
\theta_{a} \\
\phi_{a}
\end{array}\right]\left(k, l, \tau_{a}\right)
\end{aligned}
$$

$S^{(2)}$ is described in terms of the infinite matrix $I-Q$ for

$$
Q=\left[\begin{array}{cc}
0 & \xi F_{1}\left[\begin{array}{l}
\theta_{1} \\
\phi_{1}
\end{array}\right] \\
-\xi F_{2}\left[\begin{array}{l}
\theta_{2} \\
\phi_{2}
\end{array}\right] & 0
\end{array}\right], \quad \xi=\sqrt{-1}
$$

\section{Theorem 9 (Tuite-Z)}

a) The infinite matrix $(I-Q)^{-1}=\sum_{n \geq 0} Q^{n}$ is convergent for $\left(\tau_{1}, \tau_{2}, \varepsilon\right) \in \mathcal{D}^{\varepsilon}$, $\mathcal{D}^{\varepsilon}$.

\subsection{Genus Two Szegö Kernel in the $\rho$-Formalism}

It is convenient to define $\kappa \in\left[-\frac{1}{2}, \frac{1}{2}\right)$ by $\phi_{2}=-\mathrm{e}^{2 \pi i \kappa}$. Then we prove [1] the following

Theorem 10 (Tuite-Z) $S^{(2)}$ is holomorphic in $\rho$ for $|\rho|<r_{1} r_{2}$ with

$$
S^{(2)}(x, y)=S_{\kappa}^{(1)}(x, y)+O(\rho),
$$

for $x, y \in \hat{\Sigma}^{(1)}$ where $S_{\kappa}^{(1)}(x, y)$ is defined for $\kappa \neq-\frac{1}{2}$, by

Let $k_{a}=k+(-1)^{\bar{a}} \kappa$, for $a=1,2$ and integer $k \geq 1$. We introduce the moments for $S_{\kappa}^{(1)}(x, y)$ : 


$$
\begin{aligned}
G_{a b}(k, l) & =G_{a b}\left[\begin{array}{c}
\theta^{(1)} \\
\phi^{(1)}
\end{array}\right](\kappa ; k, l) \\
& =\frac{\rho^{\frac{1}{2}\left(k_{a}+l_{b}-1\right)}}{(2 \pi i)^{2}} \oint_{\mathcal{C}_{\bar{a}}\left(x_{\bar{a}}\right)} \oint_{\mathcal{C}_{b}\left(y_{b}\right)}\left(x_{\bar{a}}\right)^{-k_{a}}\left(y_{b}\right)^{-l_{b}} S_{\kappa}^{(1)}\left(x_{\bar{a}}, y_{b}\right) \mathrm{d} x_{\bar{a}}^{\frac{1}{2}} \mathrm{~d} y_{b}^{\frac{1}{2}},
\end{aligned}
$$

with associated infinite matrix $G=\left(G_{a b}(k, l)\right)$. We define also half-order differentials

$$
\begin{aligned}
& h_{a}(k, x)=h_{a}\left[\begin{array}{c}
\theta^{(1)} \\
\phi^{(1)}
\end{array}\right](\kappa ; k, x)=\frac{\rho^{\frac{1}{2}\left(k_{a}-\frac{1}{2}\right)}}{2 \pi i} \oint_{\mathcal{C}_{a}\left(y_{a}\right)} y_{a}^{-k_{a}} S_{\kappa}^{(1)}\left(x, y_{a}\right) \mathrm{d} y a_{a}^{\frac{1}{2}}, \\
& \bar{h}_{a}(k, y)=\bar{h}_{a}\left[\begin{array}{c}
\theta^{(1)} \\
\phi^{(1)}
\end{array}\right](\kappa ; k, y)=\frac{\rho^{\frac{1}{2}\left(k_{a}-\frac{1}{2}\right)}}{2 \pi i} \oint_{\mathcal{C}_{\bar{a}\left(y_{\bar{a}}\right)}} x_{\bar{a}}^{-k_{a}} S_{\kappa}^{(1)}\left(x_{\bar{a}}, y\right) \mathrm{d} x a_{\bar{a}}^{\frac{1}{2}},
\end{aligned}
$$

and let $h(x)=\left(h_{a}(k, x)\right)$ and $\bar{h}(y)=\left(\bar{h}_{a}(k, y)\right)$, denote the infinite row vectors indexed by $a, k$. From the sewing relation $z_{1} z_{2}=\rho$ we have

$$
\mathrm{d} z_{a}^{\frac{1}{2}}=(-1)^{\bar{a}} \xi \rho^{\frac{1}{2}} \frac{\mathrm{d} z_{\bar{a}}^{\frac{1}{2}}}{z_{\bar{a}}},
$$

for $\xi \in\{ \pm \sqrt{-1}\}$, depending on the branch of the double cover of $\Sigma^{(1)}$ chosen. It is convenient to define

$$
T=\xi G D^{\theta},
$$

with an infinite diagonal matrix

$$
D^{\theta}(k, l)=\left[\begin{array}{cc}
\theta^{-1} & 0 \\
0 & -\theta
\end{array}\right] \delta(k, l) .
$$

Defining $\operatorname{det}(I-T)$ by the formal power series in $\rho$

$$
\log \operatorname{det}(I-T)=\operatorname{Tr} \log (I-T)=-\sum_{n \geq 1} \frac{1}{n} \operatorname{Tr}\left(T^{n}\right),
$$

we prove in [1].

\section{Theorem 11 (Tuite-Z)}

a) $(I-T)^{-1}=\sum_{n \geq 0} T^{n}$ is convergent for $|\rho|<r_{1} r_{2}$,

b) $\operatorname{det}(I-T)$ is non-vanishing and holomorphic in $\rho$ on $\mathcal{D}^{\rho}$.

Theorem 12 (Tuite-Z) $S^{(2)}(x, y)$ is given by

$$
S^{(2)}(x, y)=S_{\kappa}^{(1)}(x, y)+\xi h(x) D^{\theta}(I-T)^{-1} \bar{h}^{T}(y) .
$$

\section{Genus One Partition and $\boldsymbol{n}$-Point Functions}

\subsection{The Torus Partition Function for a Heisenberg VOA}

For a VOA $V=\oplus_{n \geq 0} V_{n}$ of central charge $c$ define the genus one partition (trace or characteristic) function by

$$
Z_{V}^{(1)}(q)=\operatorname{Tr}_{V}\left(q^{L(0)-C / 24}\right)=\sum_{n \geq 0} \operatorname{dim} V_{n} q^{n-C / 24},
$$

for the Heisenberg VOA $M$ commutation relations with modes

$$
\begin{aligned}
& {[a(m), a(n)]=m \delta_{m,-n},} \\
& Z_{M}^{(1)}(q)=\frac{1}{\eta(\tau)} \text { for } \eta(\tau)=q^{\frac{1}{24}} \prod_{n \geq 1}\left(1-q^{n}\right) .
\end{aligned}
$$

\subsection{Genus One Twisted Graded Dimension}

We define the genus one partition function for the VOSA by the supertrace

$$
\begin{aligned}
Z_{V}^{(1)}(\tau) & =\operatorname{STr}_{V}\left(q^{L(0)-\frac{1}{24}}\right)=\operatorname{Tr}_{V}\left(\sigma q^{L(0)-\frac{1}{24}}\right) \\
& =q^{-\frac{1}{24}} \prod_{n \geq 0}\left(1-q^{n+\frac{1}{2}}\right)^{2}
\end{aligned}
$$

where $\sigma u=\mathrm{e}^{2 \pi i w t(u)} u$.

More generally, we can construct a $\sigma g$-twisted module $M_{\sigma g}$ for any automorphism $g=\mathrm{e}^{2 \pi i \beta a(0)}$ generated by the Heisenberg state $a \in V_{1}$. We introduce the second automorphism $h=\mathrm{e}^{2 \pi i \alpha a(0)}$ and define the orbifold $\sigma g$-twisted trace by

$$
Z_{V}^{(1)}\left[\begin{array}{l}
h \\
g
\end{array}\right](q)=\operatorname{STr}_{M_{\sigma g}}\left(h q^{L(0)-\frac{1}{24}}\right),
$$

to find for $\theta=\mathrm{e}^{-2 \pi i \alpha}$,

$$
\begin{aligned}
& Z_{V}^{(1)}\left[\begin{array}{l}
h \\
g
\end{array}\right](q) \\
& =q^{(\beta+1 / 2)^{2} / 2-1 / 24} \prod_{l \geq 1}\left(1-\theta^{-1} q^{l-\beta-1}\right)\left(1-\theta q^{l+\beta}\right) .
\end{aligned}
$$




\subsection{Genus One Fermionic One-Point Functions}

Each orbifold 1-point function can found from a generalized Zhu reduction formulas as a determinant.

Theorem 13 (Mason-Tuite-Z) For a Fock vector

$$
\begin{aligned}
& \boldsymbol{\Psi}[\boldsymbol{k}, \boldsymbol{l}]=\psi^{+}\left[-k_{1}\right] \cdots \psi^{+}\left[-k_{n}\right] \psi^{-}\left[-l_{1}\right] \cdots \psi^{-}\left[-l_{n}\right] \mathbf{1}, \\
& Z_{V}^{(1)}\left[\begin{array}{l}
h \\
g
\end{array}\right](\boldsymbol{\Psi}[\boldsymbol{k}, \boldsymbol{l}], q)=\operatorname{det}\left(\boldsymbol{C}\left[\begin{array}{l}
\theta \\
\varphi
\end{array}\right]\right) Z_{V}^{(1)}\left[\begin{array}{l}
h \\
g
\end{array}\right](q),
\end{aligned}
$$$$
\begin{aligned}
Z_{V}^{(1)}\left[\begin{array}{l}
h \\
g
\end{array}\right]\left(\left(v_{1}, z_{1}\right), \cdots,\left(v_{n}, z_{n}\right) ; q\right) & \equiv \operatorname{STr}_{M_{\sigma g}}\left(h Y\left(v_{1}, z_{1}\right) \cdots Y\left(v_{n}, z_{n}\right) q^{L(0)-\frac{1}{24}}\right) \\
& =Z_{V}^{(1)}\left[\begin{array}{l}
h \\
g
\end{array}\right]\left(Y\left[v_{1}, z_{1}\right] \cdot Y\left[v_{2}, z_{2}\right] \cdots Y\left[v_{n}, z_{n}\right] \cdot \mathbf{1}, q\right)
\end{aligned}
$$

where for $i, j=1,2, \cdots, n$

$$
C\left[\begin{array}{l}
\theta \\
\phi
\end{array}\right](i, j)=C\left[\begin{array}{l}
\theta \\
\phi
\end{array}\right]\left(k_{i}, l_{j}, \tau\right) .
$$

\subsection{Genus One $\boldsymbol{n}$-Point Functions for VOA}

In general, we can define the genus one orbifold $n$-point function for $v_{1}, \cdots, v_{n} \in V$ by
Every orbifold n-point function can be computed using generalized Zhu reduction formulas in terms of a determinant with entries arising from the basic 2-point function for $\psi^{+}, \psi^{-}[19]$.

\subsection{Zhu Reduction Formula}

To reduce an $n+1$-point function to a sum of $n$-point functions we need:

The supertrace property

$$
\begin{aligned}
& \operatorname{STr}(A B)=p(A, B) \mathrm{S} \operatorname{Tr}(B A), \\
& p(A, B)=(-1)^{p(A) p(B),}
\end{aligned}
$$

Borcherds commutation formula:

$$
[a(m), Y(b, z)]=\sum_{j \geq 0}\left(\begin{array}{c}
m \\
j
\end{array}\right) Y(a(j) b, z) z^{m-j},
$$

expansions for $P_{k}$-functions:

$$
\begin{aligned}
& P_{1}\left[\begin{array}{l}
\theta \\
\phi
\end{array}\right]\left(z_{1}-z_{2}, \tau\right) \\
& =\frac{1}{z_{1}-z_{2}}+\sum_{k, l \geq 1} C\left[\begin{array}{l}
\theta \\
\phi
\end{array}\right](k, l) z_{1}^{k-1} z_{2}^{l-1}, \\
& P_{k}\left[\begin{array}{l}
\theta \\
\phi
\end{array}\right](z, \tau)=\frac{(-1)^{k}}{(k-1) !} \sum_{n \in \mathbb{Z}+\lambda} \frac{n^{k-1} q_{z}^{n}}{1-\theta^{-1} q^{n}} .
\end{aligned}
$$

Theorem 14 (Mason-Tuite-Z) For any $v_{1}, \cdots, v_{n} \in V$ we have

$$
\begin{aligned}
Z^{(1)}\left(v, v_{1}, \cdots v_{n} ; \tau\right) & =\sum_{r=1}^{n} \sum_{m \geq 0} p_{1, \cdots, r-1} P_{m+1}\left[\begin{array}{l}
\theta \\
\phi
\end{array}\right]\left(z-z_{r}, \tau\right) Z^{(1)}\left(v_{1}, \cdots, v[m] v_{r}, \cdots, v_{n} ; \tau\right) \\
& +\delta_{\theta, 1 ; \phi, 1} \operatorname{STr}\left(o(v) Y_{M}\left(q_{1}^{L(0)} v_{1}, q_{1}\right) \cdots Y_{M}\left(q_{n}^{L(0)} v_{n}, q_{n}\right) q^{L(0)-\frac{c}{24}}\right)
\end{aligned}
$$

where $p_{A, \cdots, B_{r-1}}$ is given by

$$
p\left(A, B_{1} \cdots B_{r-1}\right)= \begin{cases}1 & \text { for } r=1 \\ (-1)^{p(A)\left[p\left(B_{1}\right)+\cdots+p\left(B_{r-1}\right)\right]} & \text { for } r>1 .\end{cases}
$$

\subsection{General Genus One Fermionic $n$-Point} Functions

The generating two-point function (for $(\theta, \phi) \neq(1,1))$ is given by

$$
Z_{V}^{(1)}\left[\begin{array}{l}
h \\
g
\end{array}\right]\left(\left(\psi^{+}, z_{1}\right),\left(\psi^{-}, z_{2}\right) ; q\right)=P_{1}\left[\begin{array}{l}
\theta \\
\phi
\end{array}\right]\left(z_{1}-z_{2}, q\right) Z_{V}^{(1)}\left[\begin{array}{l}
h \\
g
\end{array}\right](q)
$$

\section{Theorem 15 (Mason-Tuite-Z)}

$$
Z_{V}^{(1)}\left[\begin{array}{l}
h \\
g
\end{array}\right]\left(\left(v_{1}, z_{1}\right), \cdots,\left(v_{n}, z_{n}\right) ; q\right)=\operatorname{det} M Z_{V}^{(1)}\left[\begin{array}{l}
h \\
g
\end{array}\right](q) \text {. }
$$

Theorem 16 (Mason-Tuite-Z) For $n$ Fock vectors

$$
\boldsymbol{\Psi}^{(a)}=\boldsymbol{\Psi}^{(a)}\left[-\boldsymbol{k}^{(a)} ;-\mathbf{l}^{(a)}\right]
$$

and

$$
\boldsymbol{\Psi}_{h}^{(a)}=\boldsymbol{\Psi}^{(a)}\left[-\boldsymbol{k}^{(a)} ;-\mathbf{l}^{(a)}\right]_{h}
$$


for $\boldsymbol{k}^{(a)}=k_{1}^{(a)}, \cdots, k_{s_{a}}^{(a)}$ and $\mathbf{l}^{(a)}=l_{1}^{(a)}, \cdots, l_{t_{a}}^{(a)}$ with $a=1, \cdots, n$. Then for $(\theta, \phi) \neq(1,1)$ the corresponding $n$-point functions are non-vanishing provided

$$
\sum_{a=1}^{n}\left(s_{a}-t_{a}\right)=0
$$

and

$$
\begin{aligned}
& Z_{V}^{(1)}\left[\begin{array}{l}
f \\
g
\end{array}\right]\left(\left(\boldsymbol{\Psi}^{(1)}, z_{1}\right), \cdots,\left(\boldsymbol{\Psi}^{(n)}, z_{n}\right) ; \tau\right) \\
& =\varepsilon \operatorname{det} \boldsymbol{M} \cdot Z_{V}^{(1)}\left[\begin{array}{l}
f \\
g
\end{array}\right](\tau),
\end{aligned}
$$

where $\varepsilon$ is certain parity factor. Here $\boldsymbol{M}$ is the block matrix

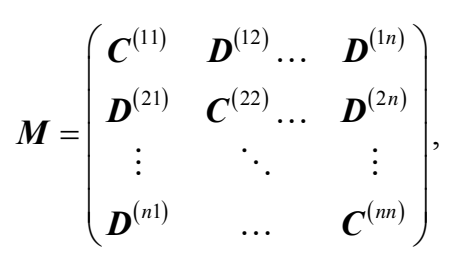

with

$$
\begin{aligned}
& Z^{(1)}\left[\begin{array}{l}
f_{1} \\
g_{1}
\end{array}\right]\left(u, z_{2} ; v_{1}, x_{1} ; \cdots ; v_{n}, x_{n} ; \bar{u}, z_{1} ; \tau\right) \\
& \equiv \operatorname{STr}_{V_{\sigma g_{1}}}\left(f_{1} \mathcal{Y}\left(q_{z_{2}}^{L_{\sigma g_{2}}(0)} u, q_{z_{2}}\right) Y\left(q_{1}^{L(0)} v_{1}, q_{1}\right) \cdots Y\left(q_{n}^{L(0)} v_{n}, q_{n}\right) \mathcal{Y}\left(q_{z_{1}}^{L_{\sigma-1}^{-1}(0)} \bar{u}, q_{z_{1}}\right) q^{L_{\sigma g_{1}}(0)-c / 24}\right),
\end{aligned}
$$

where $q=\exp (2 \pi i \tau), \quad q_{k}=\exp \left(x_{k}\right), \quad q_{z_{j}}=\exp \left(z_{j}\right)$, $j=1,2 ; 1 \leq k \leq n$, for variables $x_{1}, \cdots, x_{n}$ associated to the local coordinates on the torus, and $\bar{u}$ is dual for $u$ with respect to the invariant form on $V_{\sigma g_{2}}$. The super-

$$
\boldsymbol{C}^{(a a)}(i, j)=C\left[\begin{array}{l}
\theta \\
\phi
\end{array}\right]\left(k_{i}^{(a)}, l_{j}^{(a)}, \tau\right),\left(1 \leq i \leq s_{a}, 1 \leq j \leq t_{a}\right),
$$

for $s_{a}, t_{a} \geq 1$ with $1 \leq a \leq n$ and

$$
\boldsymbol{D}^{(a b)}(i, j)=D\left[\begin{array}{l}
\theta \\
\phi
\end{array}\right]\left(k_{i}^{(a)}, l_{j}^{(b)}, \tau, z_{a b}\right),\left(1 \leq i \leq s_{a}, 1 \leq j \leq t_{b}\right),
$$

for $s_{a}, t_{b} \geq 1$ with $1 \leq a, b \leq n$ and $a \neq b . \varepsilon$ is the sign of the permutation associated with the reordering of $\psi^{ \pm}$to the alternating ordering.

Furthermore, the $n$-point function is an analytic function in $z_{a}$ and converges absolutely and uniformly on compact subsets of the domain $|q|<\left|q_{z_{a b}}\right|<1$.

\subsection{Torus Intertwined $\boldsymbol{n}$-Point Functions}

As in ordinary (non-intertwined) case [2,19,20,24-29] we construct in [4] the partition and $n$-point functions [30-39] for vertex operator algebra modules.

Let $g_{i}, f_{i}, i=1,2$ be VOSA $V$ automorphisms commuting with $\sigma v=(-1)^{p(v)} v$. For $u \in V_{\sigma g_{2}}$ and the states $v_{1}, \cdots, v_{n} \in V$ we define the intertwined $n$-point function [4] on the torus by

$$
\begin{aligned}
& \mathcal{F}^{(1)}\left[\begin{array}{c}
f_{1} \\
g_{1}
\end{array}\right]\left(u, z_{2} ; v_{1}, x_{1} ; \cdots ; v_{n}, x_{n} ; \bar{u}, z_{1} ; \tau\right) \\
& \equiv Z^{(1)}\left[\begin{array}{c}
f_{1} \\
g_{1}
\end{array}\right]\left(u, z_{2} ; v_{1}, x_{1} ; \cdots ; v_{n}, x_{n} ; \bar{u}, z_{1} ; \tau\right) \mathrm{d} z_{2}^{w t[u]} \mathrm{d} z_{1}^{w t[\bar{u}]} \prod_{i=1}^{n} \mathrm{~d} x_{i}^{w t\left[v_{i}\right]},
\end{aligned}
$$

associated to the torus intertwined $n$-point function.

\subsection{Torus Intertwined Two-Point Function}

The rank two free fermionic VOSA $V\left(H, \mathbb{Z}+\frac{1}{2}\right)^{\otimes 2},[10]$ is generated by $\psi^{ \pm}$with

$$
\begin{aligned}
& {\left[\psi^{+}(m), \psi^{-}(n)\right]=\delta_{m,-n-1},\left[\psi^{+}(m), \psi^{+}(n)\right]=0,} \\
& {\left[\psi^{-}(m), \psi^{-}(n)\right]=0 .}
\end{aligned}
$$

trace over a $V$-module $N$ is defined by

$$
\operatorname{STr}_{N}(X)=\operatorname{Tr}_{N}(\sigma X) \text {. }
$$

For an element $u \in V_{\sigma g_{2}}$ of a VOSA $g$-twisted $V$-module we introduce also the differential form
The rank two free fermion VOSA intertwined torus $n$-point function is parameterized by $\theta_{1}=-\mathrm{e}^{-2 \pi i \beta_{1}}$, $\phi_{1}=-\mathrm{e}^{2 \pi i \alpha_{1}}$, and $\phi_{2}=-\mathrm{e}^{-2 \pi i \kappa},[2,4]$ where

$$
\sigma f_{1}=\mathrm{e}^{2 \pi i \beta_{1} a(0)}, \sigma g_{1}=\mathrm{e}^{-2 \pi i \alpha_{1} a(0)}, \sigma g_{2}=\mathrm{e}^{2 \pi i \kappa a(0)},
$$

for real valued $\alpha_{1}, \beta_{1}, \kappa,\left(\theta_{1}, \phi_{1}\right) \neq(1,1)$.

For $u=1 \otimes \mathrm{e}^{\kappa} \equiv \mathrm{e}^{\kappa} \in V_{\sigma g_{2}}$ and $\quad v_{i}=1, \quad i=1, \cdots, n$ we obtain [4] the basic intertwined two-point function on the torus 


$$
Z^{(1)}\left[\begin{array}{l}
f_{1} \\
g_{1}
\end{array}\right]\left(\mathrm{e}^{\kappa}, z_{2} ; \mathrm{e}^{-\kappa}, z_{1} ; \tau\right) \equiv \operatorname{STr}_{V_{\sigma g_{1}}}\left(f_{1} \mathcal{Y}\left(q_{z_{2}}^{L(0)} \mathrm{e}^{\kappa}, q_{z_{2}}\right) \mathcal{Y}\left(q_{z_{1}}^{L(0)} \mathrm{e}^{-\kappa}, q_{z_{1}}\right) q^{L_{\sigma g_{1}}(0)-c / 24}\right)
$$

We then consider the differential form

$$
\mathcal{G}_{n}^{(1)}\left[\begin{array}{l}
f_{1} \\
g_{1}
\end{array}\right]\left(x_{1}, y_{1}, \cdots, x_{n}, y_{n}\right) \equiv \mathcal{F}^{(1)}\left[\begin{array}{l}
f_{1} \\
g_{1}
\end{array}\right]\left(\mathrm{e}^{\kappa}, w ; \psi^{+}, x_{1} ; \psi^{-}, y_{1} ; \cdots ; \psi^{+}, x_{n} ; \psi^{-}, y_{n} ; \mathrm{e}^{-\kappa}, 0 ; \tau\right),
$$

associated to the torus intertwined $2 n$-point function

$$
Z^{(1)}\left[\begin{array}{l}
f_{1} \\
g_{1}
\end{array}\right]\left(\mathrm{e}^{\kappa}, w ; \psi^{+}, x_{1} ; \psi^{-}, y_{1} ; \cdots ; \psi^{+}, x_{n} ; \psi^{-}, y_{n} ; \mathrm{e}^{-\kappa}, 0 ; \tau\right)
$$

with alternatively inserted $n$ states $\psi^{+}$and $n$ states $\psi^{-}$distributed on the resulting genus two Riemann surface $\Sigma^{(2)}$ at points $x_{i}, y_{i} \in \Sigma^{(2)}, i=1, \cdots, n$. We then prove in [4].

Theorem 17 (Tuite-Z) For the rank two free fermion vertex operator superalgebra $V$ and for $(\theta, \phi) \neq(1,1)$ the generating form is given by

$$
\begin{aligned}
& \mathcal{G}_{n}^{(1)}\left[\begin{array}{l}
f_{1} \\
g_{1}
\end{array}\right]\left(x_{1}, y_{1}, \cdots, x_{n}, y_{n}\right)=Z^{(1)}\left[\begin{array}{l}
f_{1} \\
g_{1}
\end{array}\right]\left(\mathrm{e}^{\kappa}, w ; \mathrm{e}^{-\kappa}, 0 ; \tau\right) \operatorname{det} S_{\kappa}^{(1)}, \\
& Z^{(1)}\left[\begin{array}{l}
f_{1} \\
g_{1}
\end{array}\right]\left(\mathrm{e}^{\kappa}, w ; \mathrm{e}^{-\kappa}, 0 ; \tau\right)=\frac{1}{\eta(\tau)} \frac{\vartheta^{(1)}\left[\begin{array}{c}
\alpha_{1} \\
\beta_{1}
\end{array}\right](\kappa w, \tau)}{K^{(1)}(w, \tau)^{\kappa^{2}}},
\end{aligned}
$$

is the basic intertwined two-point function on the torus, and $n \times n-$ matrix

$$
S_{\kappa}^{(1)}=\left[S_{\kappa}^{(1)}\left[\begin{array}{l}
\theta_{1} \\
\phi_{1}
\end{array}\right]\left(x_{i}, y_{j} \mid \tau, \omega\right)\right], i, j=1, \cdots n,
$$

with elements given by parts of the Szegö kernel.

\section{Genus Two Partition and $n$-Point Functions}

\subsection{Genus Two Partition Function in $\varepsilon$-Formalism}

We define the genus two partition function in the earlier sewing scheme in terms of data coming from the two tori, namely the set of 1-point functions $Z_{V}^{(1)}\left(u, \tau_{a}\right)$ for all $u \in V$. We assume that $V$ has a nondegenerate invariant bilinear form - the Li-Zamolodchikov metric. Define

$$
Z_{V}^{(2)}\left(\tau_{1}, \tau_{2}, \varepsilon\right)=\sum_{n \geq 0} \varepsilon^{n} \sum_{u \in V_{[n]}} Z_{V}^{(1)}\left(u, \tau_{1}\right) Z_{V}^{(1)}\left(\bar{u}, \tau_{2}\right) .
$$

The inner sum is taken over any basis and $\bar{u}$ is dual to $u$ wrt to the Li-Zamolodchikov metric.

\subsection{Genus Two Partition Function for the Heisenberg VOA}

We can compute $Z_{M}^{(2)}$ using a combinatorial-graphical technique based on the explicit Fock basis and recalling the infinite matrices $A_{1}, A_{2}$.

Theorem 18 (Mason-Tuite) a) The genus two partition function for the rank one Heisenberg VOA is

$$
Z_{M}^{(2)}\left(\tau_{1}, \tau_{2}, \varepsilon\right)=\frac{1}{\eta\left(\tau_{1}\right) \eta\left(\tau_{2}\right)}\left(\operatorname{det}\left(I-A_{1} A_{2}\right)\right)^{-1 / 2}
$$

b) $Z_{M}^{(2)}\left(\tau_{1}, \tau_{2}, \varepsilon\right)$ is holomorphic on the domain $\mathcal{D}^{\varepsilon}$

c) $Z_{M}^{(2)}\left(\tau_{1}, \tau_{2}, \varepsilon\right)^{2}$ is automorphic of weight -1 ;

d) $Z_{M}^{(2)}\left(\tau_{1}, \tau_{2}, \varepsilon\right)$ has an infinite product formula.

\subsection{Genus Two Fermionic Partition Function}

Following the definition for the bosonic VOA we define for $h_{a}, g_{a}$

$$
\begin{aligned}
& Z^{(2)}\left[\begin{array}{l}
h \\
g
\end{array}\right]\left(q_{1}, q_{2}, \varepsilon\right) \\
& =\sum_{m \in \frac{1}{2} \mathbb{Z}} \varepsilon^{m} \sum_{u \in V_{[m]}} Z^{(1)}\left[\begin{array}{l}
h_{1} \\
g_{1}
\end{array}\right]\left(u, q_{1}\right) Z^{(1)}\left[\begin{array}{l}
h_{2} \\
g_{2}
\end{array}\right]\left(\bar{u}, q_{2}\right) .
\end{aligned}
$$

The inner sum is taken over any $V_{[m]}$ basis and $\bar{u}$ is dual to $u$ with respect to the $\mathrm{Li}$-Zamolodchikov square 
bracket metric. $Z_{V}^{(1)}\left[\begin{array}{l}h_{a} \\ g_{a}\end{array}\right]\left(u, q_{a}\right)$ is the genus one orbifold 1-point function. Recall that the non-zero 1-point functions arise for Fock vectors

$$
\begin{aligned}
& \boldsymbol{\Psi}[\boldsymbol{k}, \boldsymbol{l}] \\
& =\psi^{+}\left[-k_{1}\right] \cdots \psi^{+}\left[-k_{n}\right] \psi^{-}\left[-l_{1}\right] \cdots \psi^{-}\left[-l_{n}\right] \mathbf{1},
\end{aligned}
$$

such that

$$
\begin{aligned}
& m=w t \boldsymbol{\Psi}[\boldsymbol{k}, \boldsymbol{l}]=\sum_{1 \leq i \leq n}\left(k_{i}+l_{i}+1\right), \\
& Z_{V}^{(1)}\left[\begin{array}{l}
h \\
g
\end{array}\right](\boldsymbol{\Psi}[\boldsymbol{k}, \boldsymbol{l}], q)=\operatorname{det}\left(\boldsymbol{C}\left[\begin{array}{l}
\theta \\
\phi
\end{array}\right]\right) Z_{V}^{(1)}\left[\begin{array}{l}
h \\
g
\end{array}\right](q) .
\end{aligned}
$$

The Li-Zamolodchikov metric dual to the Fock vector is

$$
\overline{\boldsymbol{\Psi}}[\boldsymbol{k}, \boldsymbol{l}]=(-1)^{n} \boldsymbol{\Psi}[\boldsymbol{l}, \boldsymbol{k}] .
$$

Recalling the infinite matrix $Q$ we find Theorem 19 (Tuite-Z)

a) The genus two orbifold partition function is

$$
\begin{aligned}
& Z^{(2)}\left[\begin{array}{l}
h \\
g
\end{array}\right]\left(q_{1}, q_{2}, \varepsilon\right) \\
& =Z^{(1)}\left[\begin{array}{l}
h_{1} \\
g_{1}
\end{array}\right]\left(q_{1}\right) Z^{(1)}\left[\begin{array}{l}
h_{2} \\
g_{2}
\end{array}\right]\left(q_{2}\right) \operatorname{det}(I-Q) ;
\end{aligned}
$$

b) $Z^{(2)}\left[\begin{array}{l}h \\ g\end{array}\right]\left(q_{1}, q_{2}, \varepsilon\right)$ is holomorphic on the domain $\mathcal{D}^{\varepsilon} ;$

c) $Z^{(2)}\left[\begin{array}{l}h \\ g\end{array}\right]\left(q_{1}, q_{2}, \varepsilon\right)$ has natural modular properties under the action of $G$.

\subsection{Genus Two Partition and $\boldsymbol{n}$-Point Functions in $\rho$-Formalism}

Let $f_{i}, i=1,2$ be automorphisms, and $V_{\sigma g_{j}}$ be twisted $V$-modules of a vertex operator superalgebra $V$. For $x_{1}, \cdots, x_{n} \in \Sigma^{(1)}$ with $\left|x_{k}\right| \geq|\rho| / r_{2}$ and $\left|x_{k}-w\right| \geq|\rho| / r_{1}$, $k=1, \cdots, n$, we define the genus two $n$-point function [4] in the $\rho$-formalism by

$$
Z^{(2)}\left[\begin{array}{l}
f \\
g
\end{array}\right]\left(v_{1}, x_{1} ; \cdots ; v_{n}, x_{n} ; \tau, w, \rho\right)=\sum_{k \geq 0 u \in V_{\sigma g_{2}}[k]} \rho^{k} Z^{(1)}\left[\begin{array}{c}
f_{1} \\
g_{1}
\end{array}\right]\left(u, w+z_{2} ; v_{1}, x_{1} ; \cdots ; v_{n}, x_{n} ; f_{2} \bar{u}, z_{1} ; \tau\right) \text {, }
$$

where $(f, g)=\left(\left(f_{i}\right),\left(g_{i}\right)\right)$, where $f$ (respectively $g$ ) denotes the pair $f_{1}, f_{2}$ (respectively $g_{1}, g_{2}$ ). The sum is taken over any $V_{\sigma g_{2}}$-basis.

In particular, we introduce the genus two partition function

$$
Z^{(2)}\left[\begin{array}{l}
f \\
g
\end{array}\right](\tau, w, \rho)=\sum_{u \in V_{\sigma g_{2}}} Z^{(1)}\left[\begin{array}{l}
f_{1} \\
g_{1}
\end{array}\right]\left(u, w ; f_{2} \bar{u}, 0 ; \tau\right)
$$

where $Z^{(1)}\left[\begin{array}{l}f_{1} \\ g_{1}\end{array}\right]\left(u, w ; f_{2} \bar{u}, 0 ; \tau\right)$ is the genus one inter-

twined two point function.

Remark 1 We can generalize the genus two n-point function by introducing and computing the differential form associated to the torus $n$-point function containing several intertwining operators in the supertrace as well as corresponding genus two $n$-point functions.

Similar to the ordinary genus two case [2], we define the differential form [4] associated to the $n$-point function on a sewn genus two Riemann surface for $v_{i} \in V$ and $x_{i} \in \Sigma^{(2)}, i=1, \cdots, n$ with

$$
\left|x_{i}\right| \geq|\rho| / r_{2}, \quad\left|x_{i}-w\right| \geq|\rho| / r_{1},
$$

$$
\mathcal{F}^{(2)}\left[\begin{array}{l}
f \\
g
\end{array}\right]\left(v_{1}, \cdots, v_{n} ; \tau, w, \rho\right) \equiv Z^{(2)}\left[\begin{array}{l}
f \\
g
\end{array}\right]\left(v_{1}, x_{1} ; \cdots ; v_{n}, x_{n} ; \tau, w, \rho\right) \prod_{i=1}^{n} \mathrm{~d} x_{i}^{w\left[t v_{i}\right]} .
$$

\section{Generalizations of Classical Identities}

\subsection{Bosonization}

The genus one orbifold partition function can be alternatively computed by decomposing the VOSA into
Heisenberg modules $M \otimes \mathrm{e}^{m}$ indexed by $a(0)$ integer eigenvalues $m$, i.e., a $\mathbb{Z}$ lattice [26]. Let $\alpha_{1}, \cdots, \alpha_{n} \in L$ be lattice elements of the rank one even lattice, $\alpha_{1}+\cdots+\alpha_{n}=0$, and $\varepsilon\left(\alpha, \alpha^{\prime}\right)$-cocycle. Then

Theorem 20 (Tuite-Mason)

$$
Z_{M}^{(1)}\left(\mathrm{e}^{\alpha_{1}}, z_{1} ; \cdots ; \mathrm{e}^{\alpha_{n}}, z_{n} ; q\right)=\frac{q^{(\beta, \beta) / 2}}{\eta(\tau)} \prod_{1 \leq r \leq n} \mathrm{e}^{\left(\left(\beta, \alpha_{r}\right) z_{r}\right)} \prod_{1 \leq i<j \leq n} \varepsilon\left(\alpha_{i}, \alpha_{j}\right) K^{(1)}\left(z_{i j}, \tau\right)^{\left(\alpha_{i}, \alpha_{j}\right)} .
$$


Then ther genus one twisted partition function is given by

$$
\begin{aligned}
Z\left[\begin{array}{l}
h \\
g
\end{array}\right](\tau) & =\sum_{m \in \mathbb{Z}}(-1)^{m} \mathrm{e}^{2 \pi i m \alpha} \operatorname{Tr}_{M \otimes \mathrm{e}^{m}}\left(q^{L(0)+\frac{1}{2}\left(\beta+\frac{1}{2}\right)^{2}-\left(\beta+\frac{1}{2}\right) m-\frac{1}{24}}\right) \\
& =\frac{\mathrm{e}^{2 \pi i(\alpha+1 / 2)(\beta+1 / 2)}}{\eta(\tau)} \vartheta\left[\begin{array}{c}
-\beta+\frac{1}{2} \\
\alpha+\frac{1}{2}
\end{array}\right](\tau) .
\end{aligned}
$$

Comparing to the fermionic product formula we obtain the classical Jacobi triple product formula:

$$
\begin{aligned}
& \prod_{n>0}\left(1-q^{n}\right)\left(1+z q^{n-1}\right)\left(1+z^{-1} q^{n}\right) \\
& =\sum_{m \in \mathbb{Z}} z^{m} q^{m(m-1) / 2} .
\end{aligned}
$$

\subsection{Genus Two Jacobi Triple Product Formula}

The genus two partition function can similarly be computed in the bosonized formalism to obtain a genus two version of the Jacobi triple product formula for the genus two Riemann theta function [19]

$$
\begin{aligned}
& Z^{(2)}\left[\begin{array}{l}
h \\
g
\end{array}\right]\left(q_{1}, q_{2}, \varepsilon\right) \\
& =\Theta^{(2)}\left[\begin{array}{l}
a \\
b
\end{array}\right]\left(\Omega^{(2)}\right) Z_{M}^{(2)}\left(q_{1}, q_{2}, \varepsilon\right),
\end{aligned}
$$

for an appropriate character valued genus two Riemann theta function

$$
\begin{aligned}
& \Theta^{(2)}\left[\begin{array}{l}
a \\
b
\end{array}\right]\left(\Omega^{(2)}\right) \\
& =\sum_{m \in \mathbb{Z}^{2}} \mathrm{e}^{i \pi(m+a) \cdot \Omega^{(2)} \cdot(m+a)+2 \pi i(m+a) \cdot b} .
\end{aligned}
$$

Comparing with the fermionic result we thus find that on $\mathcal{D}^{\varepsilon}$

$$
\begin{gathered}
\frac{\Theta^{(2)}\left[\begin{array}{l}
a \\
b
\end{array}\right]\left(\Omega^{(2)}\right)}{\vartheta^{(1)}\left[\begin{array}{l}
a_{1} \\
b_{1}
\end{array}\right]\left(\tau_{1}\right) \vartheta^{(1)}\left[\begin{array}{l}
a_{2} \\
b_{2}
\end{array}\right]\left(\tau_{2}\right)} \\
=\operatorname{det}\left(I-A_{1} A_{2}\right)^{1 / 2} \operatorname{det}(I-Q) .
\end{gathered}
$$

\subsection{Fay's Trisecant Identity}

Recall Fay's trisecant identity [21]

$$
\begin{aligned}
& \frac{\theta(x-t) \theta(y-z)}{\theta(x-z) \theta(y-t)} \theta(\xi) \theta(\xi+y-x+z-t) \\
& +\frac{\theta(z-t) \theta(y-x)}{\theta(z-x) \theta(y-t)} \theta(\xi+z-x) \theta(\xi+y-t) \\
& =\theta(\xi+z-t) \theta(\xi+y-x),
\end{aligned}
$$

for $x, y, z, t \in \Sigma, \xi \in J$, where $J$ is the Jacobian of the curve.

\subsection{Bosonized Generating Function and Trisecant Identity}

In a similar fashion we can compute the general $2 n$ generating function $G_{2 n, h}^{(1)}$ in the bosonic setting to obtain:

Theorem 21 (Mason-Tuite-Z)

$$
\begin{aligned}
& G_{2 n, h}^{(1)}\left(f ; z_{1}, \cdots, z_{n} ; z_{1}^{\prime}, \cdots, z_{n}^{\prime} ; \tau\right) \\
& =\frac{\mathrm{e}^{2 \pi i(\alpha+1 / 2)(\beta+1 / 2)}}{\eta(\tau)} \vartheta^{(1)}\left[\begin{array}{c}
-\beta+\frac{1}{2} \\
\alpha+\frac{1}{2}
\end{array}\right]\left(\sum_{i=1}^{n}\left(z_{i}-z_{i}^{\prime}\right), \tau\right) \frac{\prod_{1 \leq i<j \leq n} K^{(1)}\left(z_{i}-z_{j}, \tau\right) K^{(1)}\left(z_{i}^{\prime}-z_{j}^{\prime}, \tau\right)}{\prod_{1 \leq i, j \leq n} K^{(1)}\left(z_{i}-z_{j}^{\prime}, \tau\right)} .
\end{aligned}
$$

Comparing this to fermionic expressions for $(\theta, \phi) \neq(1,1)$. we obtain the classical Frobenius elliptic function version of generalized Fay's trisecant identity
[21]:

Corollary 1 (Mason-Tuite-Z) For $(\theta, \phi) \neq(1,1)$. we have 


$$
\operatorname{det}(\boldsymbol{P})=\frac{\vartheta^{(1)}\left[\begin{array}{c}
-\beta+\frac{1}{2} \\
\alpha+\frac{1}{2}
\end{array}\right]\left(\sum_{i=1}^{n}\left(z_{i}-z_{i}^{\prime}\right), \tau\right)}{\vartheta^{(1)}\left[\begin{array}{c}
-\beta+\frac{1}{2} \\
\alpha+\frac{1}{2}
\end{array}\right](0, \tau)} \frac{\prod_{1 \leq i<j \leq n}^{(1)}\left(z_{i}-z_{j}, \tau\right) K^{(1)}\left(z_{i}^{\prime}-z_{j}^{\prime}, \tau\right)}{\prod_{1 \leq i, j \leq n} K^{(1)}\left(z_{i}-z_{j}^{\prime}, \tau\right)} .
$$

\subsection{Generalized Fay's Trisecant Identity}

We may generalize these identities using [26]. Consider the general lattice $n$-point function. We have [19], For integers $m_{i}, n_{j} \geq 0$ satisfying $\sum_{i=1}^{r} m_{i}=\sum_{j=1}^{s} n_{j}$, we have

$$
\begin{aligned}
& Z_{V}^{(1)}\left(f ;\left(\mathbf{1} \otimes \mathrm{e}^{m_{1}}, z_{1}\right), \cdots\left(\mathbf{1} \otimes \mathrm{e}^{m_{r}}, z_{r}\right),\left(\mathbf{1} \otimes \mathrm{e}^{-n_{1}}, z_{1}^{\prime}\right), \cdots,\left(\mathbf{1} \otimes \mathrm{e}^{-n_{s}}, z_{s}^{\prime}\right) ; \tau\right) \\
& =\frac{\mathrm{e}^{2 \pi i(\alpha+1 / 2)(\beta+1 / 2)}}{\eta(\tau)} \vartheta^{(1)}\left[\begin{array}{c}
-\beta+\frac{1}{2} \\
\alpha+\frac{1}{2}
\end{array}\right]\left(\sum_{i=1}^{r} m_{i} z_{i}-\sum_{j=1}^{s} n_{j} z_{j}^{\prime}, \tau\right) \cdot \frac{\prod_{1 \leq i<k \leq r} K^{(1)}\left(z_{i}-z_{k}, \tau\right)^{m_{i} m_{k}} \prod_{1 \leq j<l \leq s} K^{(1)}\left(z_{j}^{\prime}-z_{l}^{\prime}, \tau\right)^{n_{j} n_{l}}}{\prod_{1 \leq i \leq r, 1 \leq j \leq s} K^{(1)}\left(z_{i}-z_{j}^{\prime}, \tau\right)^{m_{i} n_{j}}} .
\end{aligned}
$$

Comparing this to the expression for $n$-point functions we obtain a new elliptic generalization of Fay's trisecant identity:

$$
\operatorname{det}\left(\boldsymbol{M}_{\boldsymbol{D}}\right)=\frac{\vartheta^{(1)}\left[\begin{array}{c}
-\beta+\frac{1}{2} \\
\alpha+\frac{1}{2}
\end{array}\right]\left(\sum_{i=1}^{r} m_{i} z_{i}-\sum_{j=1}^{s} n_{j} z_{j}^{\prime}, \tau\right)}{\vartheta^{(1)}\left[\begin{array}{c}
-\beta+\frac{1}{2} \\
\alpha+\frac{1}{2}
\end{array}\right](0, \tau)} \cdot \frac{\prod_{1 \leq i<k \leq r} K^{(1)}\left(z_{i}-z_{k}, \tau\right)^{m_{i} m_{k}} \prod_{1 \leq j<\leq \leq s} K^{(1)}\left(z_{j}^{\prime}-z_{l}^{\prime}, \tau\right)^{n_{j} n_{l}}}{\prod_{1 \leq i \leq r, 1 \leq j \leq s} K^{(1)}\left(z_{i}-z_{j}^{\prime}, \tau\right)^{m_{i} n_{j}}} .
$$

Here $\boldsymbol{M}_{\boldsymbol{D}}$ is the block matrix

$$
\boldsymbol{M}_{\boldsymbol{D}}=\left(\begin{array}{ccc}
\boldsymbol{D}^{(11)} & \ldots & \boldsymbol{D}^{(1 s)} \\
\vdots & \ddots & \vdots \\
\boldsymbol{D}^{(r 1)} & \ldots & \boldsymbol{D}^{(r s)}
\end{array}\right)
$$

with $\boldsymbol{D}^{(a b)}$ the $m_{a} \times n_{b}$ matrix

$$
\begin{aligned}
& \boldsymbol{D}^{(a b)}(i, j)=D\left[\begin{array}{l}
\theta \\
\phi
\end{array}\right]\left(i, j, \tau, z_{a}-z_{b}^{\prime}\right), \\
& \left(1 \leq i \leq m_{a}, 1 \leq j \leq n_{b}\right),
\end{aligned}
$$

for $1 \leq a \leq r$ and $1 \leq b \leq s$, and $D$-functions are given by the expansion

$$
P_{1}\left[\begin{array}{l}
\theta \\
\phi
\end{array}\right]\left(z+z_{1}-z_{2}, \tau\right)=\sum_{k, l \geq 1} D\left[\begin{array}{l}
\theta \\
\phi
\end{array}\right](k, l, z) z_{1}^{k-1} z_{2}^{l-1} .
$$

Corollary 2 (Mason-Tuite-Z) For $(\theta, \phi) \neq(1,1)$. we have

\section{Genus Two Intertwined Partition and n-Point Functions}

In [4] we then prove:

Theorem 22 (Tuite-Z) Let $V_{\sigma g_{i}}, i=1,2$ be $\sigma g_{i}$ twisted $V$-modules for the rank two free fermion vertex operator superalgebra $V$. Let $(\theta, \phi) \neq(1,1)$. Then the partition function on a genus two Riemann surface obtained in the $\rho$-self-sewing formalism of the torus is a non-vanishing holomorphic function on $\mathcal{D}^{\rho}$ given by

$$
\begin{aligned}
& Z^{(2)}\left[\begin{array}{l}
f \\
g
\end{array}\right](\tau, w, \rho) \\
& =Z^{(1)}\left[\begin{array}{l}
f_{1} \\
g_{1}
\end{array}\right]\left(\mathrm{e}^{\kappa}, w ; \mathrm{e}^{-\kappa}, 0 ; \tau\right) b(\kappa) \operatorname{det}(1-T),
\end{aligned}
$$


where $Z^{(1)}\left[\begin{array}{l}f_{1} \\ g_{1}\end{array}\right]\left(\mathrm{e}^{\kappa}, w ; \mathrm{e}^{-\kappa}, 0 ; \tau\right)$ is the intertwined $V$ module $V_{\sigma g_{1}}$ torus basic two-point function, and $b(\kappa)$ is some function.

We may similarly compute the genus two partition function in the $\rho$-formalism for the original rank one fermion VOSA $V\left(H, \mathbb{Z}+\frac{1}{2}\right)$ in which case we can only construct a $\sigma$-twisted module. Then we have [4] the following:

Corollary 3 (Tuite-Z) Let $V$ be the rank one free fermion vertex operator superalgebra and $f_{1}, g_{1} \in\{\sigma, 1\}$, $a=1,2$, be automorphisms. Then the partition function for $V$-module $V_{\sigma g_{1}}$ on a genus two Riemann surface obtained from $\rho$ formalism of a self-sewn torus $\Sigma^{(1)}$ is given by

$$
\begin{aligned}
& Z_{\text {rank1 } 1}^{(2)}\left[\begin{array}{l}
f \\
g
\end{array}\right](\tau, w, \rho) \\
& =Z_{\text {rank1 }}^{(1)}\left[\begin{array}{l}
f_{1} \\
g_{1}
\end{array}\right]\left(\mathrm{e}^{\kappa}, w ; \mathrm{e}^{-\kappa}, 0 ; \tau\right) \operatorname{det}(I-T)^{1 / 2},
\end{aligned}
$$

where $Z_{\text {rank1 } 1}^{(1)}\left[\begin{array}{l}f_{1} \\ g_{1}\end{array}\right]\left(\mathrm{e}^{\kappa}, w ; \mathrm{e}^{-\kappa}, 0 ; \tau\right)$ is the rank one fermion intertwined partition function on the original torus.

\subsection{Genus Two Generating Form}

In [4] we define matrices

$$
\begin{gathered}
S^{(2)}=\left(S^{(2)}\left(x_{i}, y_{j}\right)\right), \quad S_{\kappa}^{(1)}=\left(S_{\kappa}^{(1)}\left(x_{i}, y_{j}\right)\right), \\
H^{+}=\left(\left(h\left(x_{i}\right)\right)(k, a)\right), H^{-}=\left(\left(\bar{h}\left(y_{i}\right)\right)(l, b)\right)^{T} .
\end{gathered}
$$

$S^{(2)}$ and $S_{\kappa}^{(1)}$ are finite matrices indexed by $x_{i}, y_{j}$ for $i, j=1, \cdots, n ; H^{+}$is semi-infinite with $n$ rows indexed by $x_{i}$ and columns indexed by $k \geq 1$ and $a=1,2$ and $H^{-}$is semi-infinite with rows indexed by $l \geq 1$ and $b=1,2$ and with $n$ columns indexed by $y_{j}$. We then prove

Lemma 2 (Tuite-Z)

$$
\operatorname{det}\left[\begin{array}{cc}
S_{\kappa}^{(1)} & \xi H^{+} D^{\theta_{2}} \\
H^{-} & I-T
\end{array}\right]=\operatorname{det} S^{(2)} \operatorname{det}(I-T),
$$

with $T, D^{\theta_{2}}$.

Introduce the differential form

$$
\begin{aligned}
& \mathcal{G}_{n}^{(2)}\left[\begin{array}{l}
f \\
g
\end{array}\right]\left(x_{1}, y_{1}, \cdots, x_{n}, y_{n}\right) \\
& =\mathcal{F}^{(2)}\left[\begin{array}{l}
f \\
g
\end{array}\right]\left(\psi^{+}, \psi^{-}, \cdots, \psi^{+}, \psi^{-} ; \tau, w, \rho\right),
\end{aligned}
$$

associated to the rank two free fermion VOSA genus two
$2 n$-point function

$$
Z^{(2)}\left[\begin{array}{l}
f \\
g
\end{array}\right]\left(\psi^{+}, x_{1} ; \psi^{-}, y_{1} ; \cdots ; \psi^{+}, x_{n} ; \psi^{-}, y_{n} ; \tau, w, \rho\right),
$$

with alternatively inserted $n$ states $\psi^{+}$and $n$ states $\psi^{-}$. The states are distributed on the genus two Riemann surface $\Sigma^{(2)}$ at points $x_{i}, y_{i} \in \Sigma^{(2)}, i=1, \cdots, n$. Then we have

Theorem 23 (Tuite-Z) All $n$-point functions for rank two free fermion VOSA twisted modules $V_{\sigma g}$ on self-sewn torus are generated by the differential form

$$
\begin{aligned}
& \mathcal{G}_{n}^{(2)}\left[\begin{array}{l}
f \\
g
\end{array}\right]\left(x_{1}, y_{1}, \cdots, x_{n}, y_{n}\right) \\
& =Z^{(2)}\left[\begin{array}{l}
f \\
g
\end{array}\right](\tau, w, \rho) \operatorname{det} S^{(2)},
\end{aligned}
$$

where the elements of the matrix

$$
S^{(2)}=\left[S^{(2)}\left[\begin{array}{l}
\theta \\
\phi
\end{array}\right]\left(x_{i}, y_{j} \mid \tau, w\right)\right], i, j=1, \cdots, n
$$

and $Z^{(2)}\left[\begin{array}{l}f \\ g\end{array}\right](\tau, w, \rho)$ is the genus two partition function.

\subsection{Modular Invariance Properties of Intertwined Functions}

Following the ordinary case $[20,27,40]$ we would like to describe modular properties of genus two "intertwined" partition and $n$-point generating functions. As in [27], consider $\hat{H} \subset \operatorname{Sp}(4, \mathbb{Z})$ with elements

$$
\mu(a, b, c)=\left(\begin{array}{cccc}
1 & 0 & 0 & b \\
a & 1 & b & c \\
0 & 0 & 1 & -a \\
0 & 0 & 0 & 1
\end{array}\right) .
$$

$\hat{H} \quad$ is generated by $A=\mu(1,0,0), B=\mu(0,1,0)$ and $C=\mu(0,0,1)$ with relations

$$
[A, B] C^{-2}=[A, C]=[B, C]=1 .
$$

We also define $\Gamma_{1} \subset S p(4, \mathbb{Z})$ where $\Gamma_{1} \cong S L(2, \mathbb{Z})$ with elements

$$
\gamma_{1}=\left(\begin{array}{cccc}
a_{1} & 0 & b_{1} & 0 \\
0 & 1 & 0 & 0 \\
c_{1} & 0 & d_{1} & 0 \\
0 & 0 & 0 & 1
\end{array}\right), a_{1} d_{1}-b_{1} c_{1}=1 .
$$

Together these groups generate

$$
L=\hat{H} \rtimes \Gamma_{1} \subset S p(4, \mathbb{Z}) .
$$

From [27] we find that $L$ acts on the domain $\mathcal{D}^{\rho}$ of 
as follows:

$$
\begin{array}{cc}
\mu(a, b, c) \cdot(\tau, w, \rho)=(\tau, w+2 \pi i a \tau+2 \pi i b, \rho), & \text { on the torus intertwined two-point function } \\
\gamma_{1} \cdot(\tau, w, \rho)=\left(\frac{a_{1} \tau+b_{1}}{c_{1} \tau+d_{1}}, \frac{w}{c_{1} \tau+d_{1}}, \frac{\rho}{\left(c_{1} \tau+d_{1}\right)^{2}}\right) . & Z^{(1)}\left[\begin{array}{c}
f_{1} \\
g_{1}
\end{array}\right](u, w ; v, 0 ; \tau) \\
Z^{(1)}\left[\begin{array}{c}
f_{1} \\
g_{1}
\end{array}\right] \mid \gamma_{1}(u, w ; v, 0 ; \tau)=Z^{(1)}\left(\gamma_{1} \cdot\left[\begin{array}{c}
f_{1} \\
g_{1}
\end{array}\right]\right)\left(u, \gamma_{1} \cdot w ; v, 0 ; \gamma_{1} \cdot \tau\right),
\end{array}
$$

We then define [4] a group action of $\gamma_{1} \in S L(2, \mathbb{Z})$ with the standard action $\gamma_{1} \cdot \tau$ and $\gamma_{1} \cdot w$, and

$$
\gamma_{1} \cdot\left[\begin{array}{l}
f_{1} \\
g_{1}
\end{array}\right]=\left[\begin{array}{ll}
f_{1}^{a_{1}} & g_{1}^{b_{1}} \\
f_{1}^{c_{1}} g_{1}^{d_{1}}
\end{array}\right]
$$

and the torus multiplier $e_{\gamma_{1}}^{(1)}\left[\begin{array}{l}f_{1} \\ g_{1}\end{array}\right] \in U(1),[1,19]$. Then we have [4]

Theorem 24 (Tuite-Z) The torus intertwined twopoint function for the rank two free fermion VOSA is a

modular form (up to multiplier) with respect to $L$

$$
\begin{aligned}
& Z^{(1)}\left[\begin{array}{l}
f_{1} \\
g_{1}
\end{array}\right] \gamma_{1}(u, w ; v, 0 ; \tau) \\
& =e_{\gamma_{1}}^{(1)}\left[\begin{array}{l}
f_{1} \\
g_{1}
\end{array}\right]\left(c_{1} \tau+d_{1}\right)^{w t u+w\left(v+\kappa^{2}\right.} Z^{(1)}\left[\begin{array}{l}
f_{1} \\
g_{1}
\end{array}\right](u, w ; v, 0 ; \tau),
\end{aligned}
$$

where $u, v \in V_{\sigma g}$.

The action of the generators $A, B$ and $C$ is given by [1]

$$
A\left[\begin{array}{c}
f_{1} \\
f_{2} \\
g_{1} \\
g_{2}
\end{array}\right]=\left[\begin{array}{c}
f_{1} \\
f_{1} f_{2} \sigma \\
g_{1} g_{2}^{-1} \sigma \\
g_{2}
\end{array}\right], B\left[\begin{array}{c}
f_{1} \\
f_{2} \\
g_{1} \\
g_{2}
\end{array}\right]=\left[\begin{array}{c}
f_{1} g_{2} \sigma \\
f_{2} g_{1} \sigma \\
g_{1} \\
g_{2}
\end{array}\right], C\left[\begin{array}{c}
f_{1} \\
f_{2} \\
g_{1} \\
g_{2}
\end{array}\right]=\left[\begin{array}{c}
f_{1} \\
f_{2} g_{2} \sigma \\
g_{1} \\
g_{2}
\end{array}\right] .
$$

In a similar way we may introduce the action of $\gamma \in L$ on the genus two partition function [4]

$$
\begin{aligned}
& Z^{(2)}\left[\begin{array}{l}
f \\
g
\end{array}\right] \mid \gamma(\tau, w, \rho) \\
& =Z^{(2)}\left(\gamma \cdot\left[\begin{array}{l}
f \\
g
\end{array}\right]\right) \gamma \cdot(\tau, w, \rho), \\
& \gamma_{1} \cdot\left[\begin{array}{c}
f_{1} \\
f_{2} \\
g_{1} \\
g_{2}
\end{array}\right]=\left[\begin{array}{c}
f_{1}^{a_{1}} g_{1}^{b_{1}} \\
f_{2} \\
f_{1}^{c_{1}} g_{1}^{d_{1}} \\
g_{2}
\end{array}\right] .
\end{aligned}
$$

We may now describe the modular invariance of the genus two partition function for the rank two free fermion VOSA under the action of $L$. Define a genus two multiplier $e_{\gamma}^{(2)}\left[\begin{array}{l}f \\ g\end{array}\right] \in U(1)$ for $\gamma \in L$ in terms of the genus one multiplier as follows

$$
e_{\gamma}^{(2)}\left[\begin{array}{l}
f \\
g
\end{array}\right]=e_{\gamma_{1}}^{(1)}\left[\begin{array}{l}
f_{1} \\
g_{1}
\end{array}\right],
$$

for the generator $\gamma_{1} \in \Gamma_{1}$. We then find [4].

Theorem 25 (Tuite-Z) The genus two partition function for the rank two VOSA is modular invariant with re- spect to $L$ with the multiplier system, i.e.,

$$
Z^{(2)}\left[\begin{array}{l}
f \\
g
\end{array}\right] \mid \gamma(\tau, w, \rho)=e_{\gamma}^{(2)}\left[\begin{array}{l}
f \\
g
\end{array}\right] Z^{(2)}\left[\begin{array}{l}
f \\
g
\end{array}\right](\tau, w, \rho) .
$$

Finally, we can also obtain modular invariance for the generating form

$$
\mathcal{G}_{n}^{(2)}\left[\begin{array}{l}
f \\
g
\end{array}\right]\left(x_{1}, y_{1}, \cdots, x_{n}, y_{n}\right),
$$

for all genus two $n$-point functions [4].

Theorem 26 (Tuite-Z) $\mathcal{G}_{n}^{(2)}\left[\begin{array}{l}f \\ g\end{array}\right]\left(x_{1}, y_{1}, \cdots, x_{n}, y_{n}\right)$, is modular invariant with respect to $L$ with a multiplier.

\section{Acknowledgements}

The author would like to express his deep gratitude to the organizers of the Conference "Algebra, Combinatorics, Dynamics and Applications", Belfast, UK, August 27-30, 2012.

\section{REFERENCES}

[1] M. P. Tuite and A. Zuevsky, "The Szegö Kernel on a Sewn Riemann Surface," Communications in Mathe- 
matical Physics, Vol. 306, No. 3, 2011, pp. 617-645.

[2] M. P. Tuite and A. Zuevsky, "Genus Two Partition and Correlation Functions for Fermionic Vertex Operator Superalgebras I," Communications in Mathematical Physics, Vol. 306, No. 2, 2011, pp. 419-447. http://dx.doi.org/10.1007/s00220-011-1258-1

[3] M. P. Tuite and A. Zuevsky, "A Generalized Vertex Operator Algebra for Heisenberg Intertwiners," Journal of Pure and Applied Algebra, Vol. 216, No. 6, 2012, pp. 1253-1492. http://dx.doi.org/10.1016/j.jpaa.2011.10.025

[4] M. P. Tuite and A. Zuevsky, "Genus Two Partition and Correlation Functions for Fermionic Vertex Operator Superalgebras II," to Appear, 2013.

[5] M. P. Tuite and A. Zuevsky, "The Bosonic Vertex Operator Algebra on a Genus $g$ Riemann Surface," RIMS Kokyuroko, Vol. 1756, No. 9, 2011, pp. 81-93.

[6] R. E. Borcherds, "Vertex Algebras, Kac-Moody Algebras and the Monster," Proceedings of the National Academy of Sciences of the United States of America, Vol. 83, No. 10, 1986, pp. 3068-3071. http://dx.doi.org/10.1073/pnas.83.10.3068

[7] C. Dong and J. Lepowsky, "Generalized Vertex Algebras and Relative Vertex Operators," Birkhäuser, Boston, 1993. http://dx.doi.org/10.1007/978-1-4612-0353-7

[8] I. Frenkel, Y. Huang and J. Lepowsky, "On Axiomatic Approaches to Vertex Operator Algebras and Modules," American Mathematical Society, Providence, Rhode Island, 1993.

[9] I. Frenkel, J. Lepowsky and A. Meurman, "Vertex Operator Algebras and the Monster," Academic Press, New York, 1988

[10] V. Kac, "Vertex Operator Algebras for Beginners," University Lecture Series, AMS, Providence, 1998.

[11] C. Dong, H. Li and G. Mason, "Twisted Representation of Vertex Operator Algebras," Mathematische Annalen, Vol. 310, No. 3, 1998, pp. 571-600. http://dx.doi.org/10.1007/s002080050161

[12] C. Dong, H. Li and G. Mason, "Simple Currents and Extensions of Vertex Operator Algebras," Communications in Mathematical Physics, Vol. 180, No. 3, 1996, pp. 671707. http://dx.doi.org/10.1007/BF02099628

[13] H. Li, "Symmetric Invariant Bilinear Forms on Vertex Operator Algebras," Journal of Pure and Applied Algebra, Vol. 96, No. 3, 1994, pp. 279-297. http://dx.doi.org/10.1016/0022-4049(94)90104-X

[14] N. Scheithauer, "Vertex Algebras, Lie Algebras and Superstrings," Journal of Algebra, Vol. 200, No. 2, 1998, pp. 363-403. http://dx.doi.org/10.1006/jabr.1997.7235

[15] H. M. Farkas and I. Kra, "Theta Constants, Riemann Surfaces and the Modular Group," Graduate Studies in Mathematics, AMS, Providence, 2001.

[16] R. C. Gunning, "Lectures on Riemann Surfaces," Princeton University Press, Princeton, 1966.

[17] A. Yamada, "Precise Variational Formulas for Abelian Differentials," Kodai Mathematical Journal, Vol. 3, No. 1, 1980, pp. 114-143. http://dx.doi.org/10.2996/kmj/1138036124
[18] D. Mumford, "Tata Lectures on Theta I and II," Birkhäuser, Boston, 1983.

[19] G. Mason, M. P. Tuite and A. Zuevsky, "Torus N-Point Functions for $\mathbb{R}$-Graded Vertex Operator Superalgebras and Continuous Fermion Orbifolds," Communications in Mathematical Physics, Vol. 283, No. 2, 2008, pp. 305342. http://dx.doi.org/10.1007/s00220-008-0510-9

[20] C. Dong, H. Li and G. Mason, "Modular-Invariance of Trace Functions in Orbifold Theory and Generalized Moonshine," Communications in Mathematical Physics, Vol. 214, No. 1, 2000, pp. 1-56. http://dx.doi.org/10.1007/s002200000242

[21] J. D. Fay, "Theta Functions on Riemann Surfaces," Lecture Notes in Mathematics, Springer-Verlag, Berlin, 1973.

[22] J. D. Fay, "Kernel Functions, Analytic Torsion and Moduli Spaces," American Mathematical Society, Providence, Rhode Island, 1992.

[23] G. Mason and M. P. Tuite, "On Genus Two Riemann Surfaces Formed from Sewn Tori," Communications in Mathematical Physics, Vol. 270, No. 3, 2007, pp. $587-$ 634. http://dx.doi.org/10.1007/s00220-006-0163-5

[24] Y. Huang, "Two-Dimensional Conformal Geometry and Vertex Operator Algebras,” Birkhäuser, Boston, 1997.

[25] A. Matsuo and K. Nagatomo, "Axioms for a Vertex Algebra and the Locality of Quantum Fields," Mathematical Society of Japan, Hongo, Bunkyo-ku, Tokio, 1999.

[26] G. Mason and M. P. Tuite, "Free Bosonic Vertex Operator Algebras on Genus Two Riemann Surfaces I", Communications in Mathematical Physics, Vol. 300, No. 3, 2010, pp. 673-713.

http://dx.doi.org/10.1007/s00220-010-1126-4

[27] G. Mason and M. P. Tuite, "Free Bosonic Vertex Operator Algebras on Genus Two Riemann Surfaces II," arXiv:1111.2264v1.

[28] G. Mason and M. P. Tuite, "Chiral N-Point Functions for Free Boson and Lattice Vertex Operator Algebras," Communications in Mathematical Physics, Vol. 235, No. 1, 2003, pp. 47-68. http://dx.doi.org/10.1007/s00220-002-0772-6

[29] Y. Zhu, "Modular Invariance of Characters of Vertex Operator Algebras," Journal of the American Mathematical Society, Vol. 9, 1996, pp. 237-302. http://dx.doi.org/10.1090/S0894-0347-96-00182-8

[30] P. di Vecchia, K. Hornfeck, M. Frau, A. Lerda and S. Sciuto, "N-String, G-Loop Vertex for the Fermionic String," Physics Letter B, Vol. 211, No. 3, 1988, pp. 301307. http://dx.doi.org/10.1016/0370-2693(88)90907-0

[31] T. Eguchi and H. Ooguri, "Chiral Bosonization on a Riemann Surface," Physics Letter B, Vol. 187, No. 1-2, 1987, pp. 127-134. http://dx.doi.org/10.1016/0370-2693(87)90084-0

[32] D. Freidan and S. Shenker, "The Analytic Geometry of Two Dimensional Conformal Field Theory," Nuclear Physics B, Vol. 281, No. 3-4, 1987, pp. 509-545. http://dx.doi.org/10.1016/0550-3213(87)90418-4

[33] M. R. Gaberdiel, Ch. A. Keller and R. Volpato, "Genus Two Partition Functions of Chiral Conformal Field Theo- 
ries," arXiv:1002.3371, 2010.

[34] M. R. Gaberdiel and R. Volpato, "Higher Genus Partition Functions of Meromorphic Conformal Field Theories," Journal of High Energy Physics, Vol. 9, No. 6, 2009, p. 48.

[35] N. Kawamoto, Y. Namikawa, A. Tsuchiya and Y. Yamada, "Geometric Realization of Conformal Field Theory on Riemann Surfaces," Communications in Mathematical Physics, Vol. 116, No. 2, 1988, pp. 247-308.

[36] F. Pezzella, " $g$-Loop Vertices for Free Fermions and Bosons," Physics Letter B, Vol. 220, No. 4, 1989, pp. 544550. http://dx.doi.org/10.1016/0370-2693(89)90784-3

[37] A. K. Raina, "Fay's Trisecant Identity and Conformal Field Theory," Communications in Mathematical Physics,
Vol. 122, No. 4, 1989, pp. 625-641. http://dx.doi.org/10.1007/BF01256498

[38] A. Tsuchiya, K. Ueno and Y. Yamada, "Conformal Field Theory on Universal Family of Stable Curves with Gauge Symmetries," Academic Press, Boston, 1989.

[39] K. Ueno, "Introduction to Conformal Field Theory with Gauge Symmetries," Geometry and Physics, Lecture Notes in Pure and Applied Mathematics, Dekker, New York, 1997, pp. 603-745.

[40] G. Mason and M. P. Tuite, "Vertex Operators and Modular Forms," In: K. Kirsten and F. Williams, Eds., A Window into Zeta and Modular Physics, Cambridge University Press, Cambridge, 2010, pp. 183-278. 